Running Head: Cognitive Modeling of Identity Leadership

\title{
Exemplifying “Us": Integrating Social Identity Theory of Leadership with Cognitive Models of Categorization
}

David K. Sewell, Timothy Ballard, and Niklas K. Steffens

School of Psychology, The University of Queensland

Final draft version submitted to The Leadership Quarterly.

Published online April 19, 2021.

Published version freely accessible until July 7, 2021, via

https://authors.elsevier.com/a/lcwms3kWknrOCb

Corresponding Author:

David K. Sewell

School of Psychology

The University of Queensland

St. Lucia, QLD 4072, AUSTRALIA

d.sewell@uq.edu.au

Tel: +61 (07) 33467629

Fax: + 61 (07) 33654466 


\section{Author Note}

Address correspondence to David K. Sewell at the School of Psychology, The University of Queensland, St. Lucia, QLD 4072, Australia. Electronic mail may be sent to d.sewell@uq.edu.au. The authors were supported by Australian Research Council Discovery Early Career Researcher Awards (DE140100772 to DKS, DE180101340 to TB, and DE180100676 to NKS). 


\begin{abstract}
Identity leadership theorizing suggests that leadership effectiveness derives from a potential leader's perceived ability to create, embody, promote, and embed a shared group identity. However, little is known about how people integrate this information to form a judgment of a leader. We use cognitive modeling to operationalize leadership judgments as exemplar-and prototype-based categorization processes. Analysis of attribute rating data for 80 highly recognizable Americans revealed that leadership judgments were well-characterized by an exemplar-based model. Judgments were based overwhelmingly on promoting shared collective interests and embedding group identity. The pattern of attribute weightings was consistent for judgments of a general leadership role (i.e., as a competent leader) as well as judgments for a specific leadership role (i.e., as an effective US president). We discuss the implications of these findings for our understanding of identity leadership as well as for integrated social-cognitive models of individuals' judgements of and responses to leaders.
\end{abstract}

Keywords: Leadership Judgments; Cognitive Modeling; Exemplar Theory; Categorization 


\section{Exemplifying "Us": Integrating Social Identity Theory of Leadership with Cognitive Models of Categorization}

Decisions about whether an individual is likely to be a competent or effective leader are a fundamental aspect of any group, organization, or society. These decisions are difficult because there are a number of dimensions on which one might evaluate a potential leader (e.g., seeing the leader as 'one of us', as charismatic, or authentic). Yet, the ability to accurately judge potential leaders is critical for society to function effectively. For example, the failure of voters to accurately judge the ability of a political candidate could result in years of governmental mismanagement. Accordingly, some of the most fundamental questions in the literature on leadership concern the attributes that distinguish leaders from non-leaders or effective from ineffective leaders (either in terms of people's perceptions of these individuals, or based on objective performance-based criteria), and the psychological processes people rely on to make these distinctions.

Theories of leadership have addressed the "what" of leadership by seeking to identify attribute dimensions (e.g., using various traditional methods such as factor analysis) that distinguish perceptions of individuals as more (or less) competent leaders (Antonakis \& Dalgas, 2009; Todorov, Mandisodza, Goren, \& Hall, 2005). Though the theories vary in the exact dimensions that they focus on—some highlight attributes that contribute to a leader's authenticity (e.g., Gardner et al., 2011), the transformational capacity (or charisma) of the leader (e.g., Bass \& Riggio, 2006), the leader's ability to instil high-quality relationships with individual followers (e.g., Graen \& Uhl-Bien, 1995), or the leader's ability to embody and promote a shared social identity among leaders (e.g., Haslam et al., 2011; Hogg et al., 2012) - they share the core assumption that possessing certain attributes promotes leadership, 
including the perception of others as competent (or good or effective) leaders. Such theories are vital for fleshing out the conceptual structure of leadership, identifying likely sources of attribute information people might rely upon when evaluating an individual as a potential leader.

While such theories clearly define the "what" of leadership, they are silent (or somewhat agnostic) about the "how" of leadership in that they do not specify how people use information about certain attribute dimensions to perceive, evaluate, and make judgments about the leadership of individuals. One way of linking these theoretical structures with assumptions about psychological processing can be found in the enduring idea that leadership evaluations are at least partly based on the outcome of a categorization process. According to this account, leadership evaluations are based on the degree to which an individual belongs in the category of "good leaders". The idea has its roots in Rosch's (1978) pioneering work on the internal structure of categories, and is perhaps most closely aligned with leadership categorization theory and the work of Lord and colleagues (e.g., Lord, Foti, \& DeVader, 1984; Lord, Foti, \& Phillips, 1982) and to a lesser degree with social identity theory of leadership (particularly its notion of leader group prototypicality; e.g., Hogg, 2001; Platow \& van Knippenberg, 2001; Turner \& Haslam, 2001). Categorization-based perspectives on leadership presuppose that the attributes possessed by an individual are compared to those of a cognitive representation of the category of effective or good leaders and potentially other relevant contrast categories (e.g., ineffective or poor leaders). The extent to which a person matches or coheres with the attributes of the "good leader" category reflects the extent to which that person is judged to be a good leader. Shondrick, Dinh, and Lord (2010) describe this in terms of a (potentially implicit) pattern matching process that results in unobserved, but highly typical attributes of the "good leader" category being conferred to a categorized 
individual. Rosch's theorizing has also been fundamental to the origins of social identity theory of leadership where researchers have presupposed that leaders are effective (and are seen as effective) to the extent they are perceived as representative of the category of a shared social identity (e.g., as 'one of us' whether the social group in question is a team, organization, nation, etc.; Hogg, 2001; Turner \& Haslam, 2001). These theories provide a way of understanding how memory retrieval processes inform leadership evaluations by showing how decisions can be driven by an underlying categorization process. The categorization process need not be explicit, and is akin to recognition memory processes, but couched in terms of whether an individual is recognized as being sufficiently similar to an established cognitive representation of the target category (e.g., of "good leaders"). Given the intuitive appeal and general success of the categorization perspective on leadership, it is perhaps surprising that one of its core assertions - that leadership judgments can be viewed as the outcome of a categorization process - has so far evaded direct test.

In this article, we introduce a computational way of assessing the descriptive adequacy of categorization-based theories of leadership by combining the well-validated conceptual structure of leadership developed in the factor analytic tradition with quantitative process models of categorization that have been developed in the cognitive psychology literature (e.g., Pothos \& Wills, 2011). The logic of our general approach can be summarized as follows: If leader decisions and evaluations rely on category information, the data should be consistent with predictions generated by a model of the categorization process. To investigate this issue, we present a formal integration of social identity theorizing of leadership with cognitive theories of categorization as a means of understanding the psychological processes that underpin leadership judgments. The use of formal psychological models enables quantitative testing of a range of assumptions about how leadership 
categories might be represented (e.g., as a collection of memory traces about specific leaders, or as a summary representation(s) of a group of individuals), the relative importance of specific attributes for judging leader effectiveness, and how relative importance might change according to contextual factors. The approach opens up the scope for testing theories, as quantitative process models provide clear mappings for relating theory and data. Indeed, such approaches are becoming increasingly popular in organization science (Ballard, Palada, Griffin, \& Neal, 2019), though they have not yet been used to investigate theories of leadership. In what follows, we discuss this model-based approach to theory testing in relation to social identity theory of leadership that proposes that leaders are perceived as effective to the extent that they are seen to embody and promote the group or social category that they are seeking to lead (Haslam et al., 2011; Hogg et al., 2012; van Knippenberg, 2011), while noting that the general approach is equally compatible with other categorization-based perspectives on leadership (e.g., Lord et al., 1984).

\section{Social Identity Theorizing of Leadership}

Social identity theorizing of leadership rests on the social identity approach, which encompasses social identity theory (Tajfel \& Turner, 1979) and self-categorization theory (Turner, Hogg, Oakes, Reicher, \& Wetherell, 1987). It proposes that individuals can derive a sense of self in terms of the idiosyncratic characteristics that make them different from other individuals (as 'I' and 'me'), but also in terms of the shared characteristics of the groups that they are a member of (as 'we' and 'us'; e.g., 'us, leadership researchers', 'us, members of team $Y^{\prime}$ '). Proponents of the theory argue that individuals' internalization of a group membership in terms of the self has meaningful consequences for their attitudinal and behavioral orientation to others (Lee, Park, \& Koo, 2015; Turner, 1982; van Dick, 2001). Such consequences include their motivation to perform on behalf of the group (Haslam, 
Powell, \& Turner, 2000; van Knippenberg, 2000), their willingness to cooperate with and help each other (Blader \& Tyler, 2009; van Dick, Grojean, Christ, \& Wieseke, 2006), and their openness to others as sources of influence (Chrobot-Mason, Gerbasi, \& Cullen-Lester, 2016; Turner, 1991).

Building on these ideas, social identity theorizing asserts that leadership is a social group process that revolves around would-be leaders' promotion of a shared sense of social identity among those they seek to influence (Hogg, 2001; Platow et al., 2015; van Knippenberg \& Hogg, 2003). The degree to which leaders are seen as embodying and promoting a shared collective identity among those they want to lead determines perceived and actual leader effectiveness (Ellemers, de Gilder, \& Haslam, 2004; Hogg, van Knippenberg, \& Rast, 2012; van Dick \& Kerschreiter, 2015; van Knippenberg, 2011).

More specifically, researchers have proposed a four-dimensional model of identity leadership (Epitropaki et al., 2017; Haslam et al., 2011; Steffens et al., 2014; van Dick et al. 2018). The theory posits that leaders are more likely to be able to influence others to the extent that they are seen (a) to act as identity entrepreneurs (i.e., by creating a shared sense of 'we' among followers; Reicher \& Hopkins, 2001; Reicher, Haslam, \& Hopkins, 2005); (b) to embody the prototype of the group (i.e., by representing those characteristics that are (ingroup-defining; Hogg, 2001; Platow \& van Knippenberg, 2001); (c) to serve as champions of the group (i.e., by advancing shared (in-)group interests rather than their personal interests or interests of other (out-)groups; Haslam \& Platow, 2001; van Knippenberg \& van Knippenberg, 2005); and (d) to act as embedders of identity (i.e., by initiating and implementing structures, activities, and events that allow the group to come together and live out a shared group membership; Haslam et al., 2011). While some of the dimensions of social 
identity leadership have a longer history and have been examined extensively, others were proposed more recently and have been subjected to less empirical examination (for recent comprehensive overviews of the development of the four dimensions, see van Dick et al., 2018, and Haslam, Reicher, and Platow, 2020). For example, by far the most extensively examined dimension is group prototypicality (i.e., embodying; Hais, Hogg, \& Duck, 1997; Hogg, 2001; Turner \& Haslam, 2001; Platow \& van Knippenberg, 2001; for a recent review, see Barreto \& Hogg, 2017; Steffens, Munt, van Knippenberg, Platow, \& Haslam, 2020). This is followed by the dimensions of identity entrepreneurship (i.e., creating; e.g., Reicher \& Hopkins 1996; 2001) and identity advancement (i.e., advancing; e.g., Haslam et al., 2001; van Knippenberg \& van Knippenberg, 2005), while identity impresarioship (i.e., embedding) is the most recent theoretical innovation (Haslam et al., 2020). Researchers have argued that these dimensions are related (as they pertain around a shared sense of 'us') but also distinct. In this regard, nested model comparisons examining the factor structure by Steffens and colleagues (2014) revealed significantly better fit to the data if the four dimensions are conceptualized as four distinct factors, rather than a combined, uni-dimensional factor.

Supporting this model, meta-analytic evidence (across correlational and experimental studies) shows that leaders' engagement in aspects of identity leadership is associated with a range of outcomes including subjective evaluations of leaders such as follower trust in and support of leaders (Barreto \& Hogg, 2017) and behavioral outcomes such as in-role and extra-role performance (Steffens et al., 2020). Furthermore, a global study conducted by van Dick and colleagues (2018) provided evidence that supports the four-factor model across 19 out of 20 countries studied. Their study also showed that leaders' engagement in identity leadership was associated with followers' job satisfaction, team identification, innovation at work, and organizational citizenship behaviors. 


\section{Integrating Social Identity Theorizing of Leadership with Categorization Theories}

The framework for understanding and measuring social identity leadership was developed within a factor analytic tradition (Steffens et al., 2014), and outlines the psychological dimensions underlying identity leadership. According to the theory, information along these dimensions is used to inform people's attitudes, judgments, and choices about potential leaders, but the process by which this occurs is not explicitly detailed (e.g., Haslam et al., 2011; Hogg, 2001; Hogg et al., 2012; Steffens et al., 2014; van Dick et al., 2018). A more detailed realization of the theory requires an explicit mapping that relates dimensional information about identity leadership to behavioral outcomes - that is, specification of the cognitive processes that transform dimensional information about the stimulus into a form that can be used to make a decision. We argue that cognitive models of categorization are well-suited to this role, as they provide a psychologically principled framework for explaining how attribute information is translated into behavior, and assessing the adequacy of assumptions about the cognitive representations that mediate this relationship. We first sketch how the concept of effective leaders postulated by social identity theorizing about leadership might be combined with categorization models. Formal mathematical details are provided further below.

To illustrate how we can make the connection between measurement theories, cognitive representations, and behavior more explicit, consider a social identity theory account of how one might decide whether an individual is, or will be seen to be an effective leader or an ineffective leader (e.g., a voter deciding on a political candidate to support). According to the theory, information about the individual is represented along four key attribute dimensions: the degree to which the individual creates a shared sense of "we" 
among followers; the degree to which the individual embodies defining characteristics or ideals of the group; the degree to which the individual advances shared interests of the group; and the degree to which the individual embeds a shared identity in material reality. Once encoded, this attribute information is used to compare the individual with cognitive representations of the relevant categories under consideration (e.g., prototypes). Note that the comparison and decision processes involved need not be conscious or deliberately performed - the core theoretical claim is that leadership evaluations draw upon information held in memory.

To clarify the details of the comparison process, we must first make assumptions about how attribute information is represented psychologically. We assume that person attributes can be represented as values along continuous dimensions, consistent with Steffens et al.'s (2014) interpretation of (social) identity theory of leadership, and in keeping with standard assumptions in the categorization literature (e.g., Nosofsky, 1986). We do, however, acknowledge that it is possible to conceptualize certain attributes as discrete "all-or-none" features rather than continuously varying. For ease of exposition, and in keeping with relevant theory (Steffens et al., 2014), we focus on continuously varying dimensions, but note that the kinds of models we describe in this article can be developed in ways that accommodate discrete-valued feature representations (e.g., Lee \& Navarro, 2002; Tversky, 1977) and combinations of discrete- and continuous-valued representations (Navarro \& Lee, 2003). We further assume that information about an individual is summarized by these person attributes, and that the individual can therefore be represented as a point in a multidimensional psychological space defined by the attribute dimensions outlined by Steffens et al. 
Having established how an individual and their attributes are represented, we must then make assumptions about how category information is represented to enable a comparison. Within the leadership literature, the most common assumption is that people rely on a summary representation of a category, usually a prototype (e.g., Lord et al., 1984; Hogg, 2001; Phillips, 1984). In the broader literature on concepts and categorization, prototypes have been varyingly defined as an ideal category member, an average category member, or a summary of modal features (Murphy, 2002). The notion of a prototype reflecting an average category member has often been used in formal evaluations of prototype theory (e.g., Nosofsky, 1992a; Minda \& Smith, 2011; Reed, 1972), and we follow this convention here. We note that there are other ways of conceptualizing prototypes - for example, as an ideal leader (van Knippenberg, 2011), or as a more abstract idealization of a category (e.g., Voorspoels, Vanpaemel, \& Storms, 2011). This is a matter we address in more detail in the Discussion. Given a prototype representation, judging whether an individual is an effective or ineffective leader involves comparing that individual's attribute values to the corresponding attribute values held by the effective leader prototype and the ineffective leader prototype. An individual will be judged to be an (in)effective leader to the extent that they are similar to the (in)effective leader prototype (e.g., Phillips, 1984). According to this view, a voter who evaluates a given candidate as most similar to an average of effective leader representations stored in memory, and less similar to an average of ineffective leader representations stored in memory, will judge the candidate to also be an effective leader. We adopt this implementation of categorization models in this article, but note that multiple category representations are not required for making a decision. For example, it is also possible to use a representation of only the effective leader category to make a choice based on whether the similarity to the effective leader prototype exceeds a pre-specified threshold. Such a formulation is sometimes used in recognition memory contexts where the critical question is 
whether a stimulus should be included in a single target category instead of requiring the observer to assign a stimulus to one of several possible categories (Nosofsky, Little, \& James, 2012; Nosofsky \& Zaki, 1998).

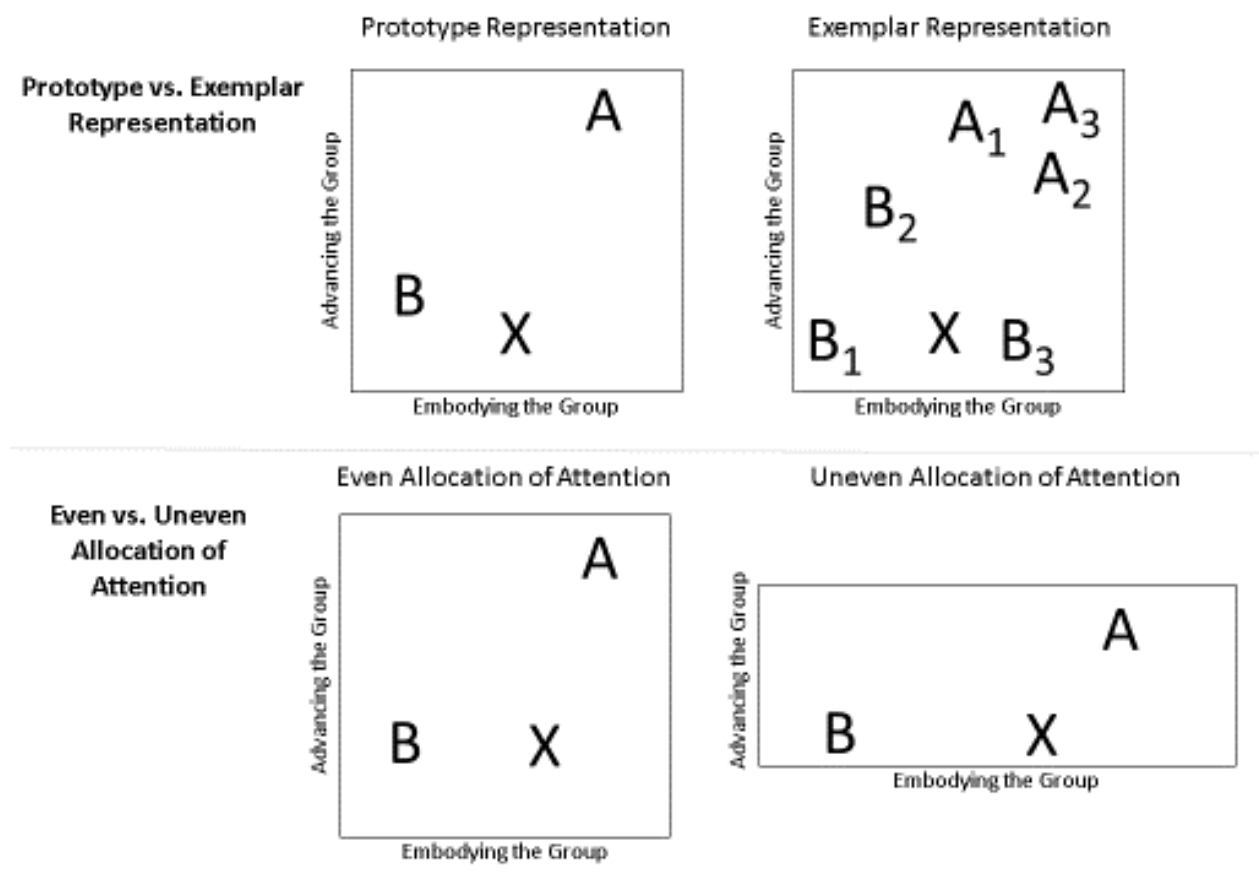

Figure 1. Visual representation of (a) prototype vs. exemplar representations of target stimuli in relation to other stimuli and (b) even vs. uneven allocation of attention to (or importance of) attribute dimensions. Top Left Panel: Geometric depiction of similarity relations between an individual, $X$, and two prototypes, $A$ and $B$, in two dimensions, defined arbitrarily as the degree to which an individual embodies the in-group (horizontal dimension) and the degree to which an individual advances the interest of the in-group (vertical dimension). The individual is most similar to the $B$ prototype, as it is spatially most proximal to it. Top Right Panel: Similarity relations between the same individual and exemplars of the two categories. The individual is most similar to exemplars from category $\mathrm{B}$, compared to exemplars from category A. Bottom Left Panel: Depiction of similarity relations between an individual, $X$, and two prototypes, $A$ and $B$, assuming an even allocation of attention across the two dimensions. Under an even allocation of attention, the individual is most similar to the $B$ prototype. Bottom Right Panel: Similarity relationship between the same individual and prototypes with an uneven allocation of attention. When attention is heavily loaded onto the "embodying" dimension (horizontal dimension), the psychological space is expanded along this dimension, and contracted along the "advancing" dimension (vertical dimension), due to limited capacity. Under this allocation of attention, the individual is most similar to the $A$ prototype despite no changes in absolute positions in psychological space.

The comparison process itself can be readily understood in geometric terms. Because both the individual and the prototype can be represented as points in a multidimensional space, similarity can be understood as reflecting the proximity of one point to another. The top left panel of Figure 1 illustrates the comparison process in a two-dimensional space for 
illustration purposes. Suppose these dimensions correspond to the degree to which a candidate embodies an in-group (horizontal dimension) and the degree to which a candidate advances the interests of the in-group (vertical dimension). Comparison of an individual candidate, $X$, with prototypes representing the categories of effective leaders, $A$, and ineffective leaders, $B$, is based on their spatial proximity to one another. In this example, the individual is closer to - more similar to - the ineffective leader prototype. The relative similarity of the individual to the two prototypes can then be used to generate behavioral predictions via a function that takes these similarity values as inputs and outputs in relation to some behavior of interest (e.g., an effectiveness rating, or a probability of categorizing the individual as an effective leader).

Prototype representation is not the only option for category representation, however. Indeed, an in-principle objection to prototype representation (or other forms of summary representations) is that they neglect the potential for representations corresponding to specific individuals to play a role in people's decisions (Medin \& Schaffer, 1978). If people retain and have access to information about individual exemplars in memory (e.g., specific individuals who are effective leaders), then one can conceive of the comparison process not as one between an individual and a prototype, but between an individual and a collection of exemplars belonging to a particular category. According to this view, a voter might decide whether to support a candidate based on that candidate's similarity to other examples of effective leaders stored in memory. Under this formulation, judging an individual's leadership effectiveness involves determining the overall similarity between that individual and exemplars belonging to the effective leader category, the $A$ s in the figure, and comparing that to the overall similarity between the individual and exemplars belonging to the ineffective leader category, the $B \mathrm{~s}$ in the figure. In each case, overall similarity is determined 
by summing the similarities between the individual and each exemplar in that category. The exemplar-based comparison process is detailed schematically in the top right panel of Figure 1. Here, the overall similarity between the individual, $X$, and the two leader categories is based on their proximity to each member of the two categories.

The foregoing illustrates how different theoretical assumptions about the way categories are represented (e.g., as prototypes or collections of exemplars) can be combined with measurement-based theories of leadership via cognitive modeling, creating readily testable integrated theories of the processes underpinning leadership judgments. A benefit of this approach is that it allows principled comparisons of the adequacy of different theoretical assumptions by examining how well the quantitative predictions of models with different category representations align with data. Indeed, sharp theoretical distinctions are possible when one theory produces a far better account of data than another. While we have focused on how such models can operate on the dimensional structure provided by social identity theory, we point out that dimensional structures posited by other theories (e.g., Epitropaki \& Martin, 2014; Offerman et al., 1994) are also amenable to the same kind of model-based investigation (e.g., by replacing the factor structure of Steffens et al., 2014, with that of say Epitropaki \& Martin, 2014). The modeling framework is sufficiently flexible to accommodate expansion (or contraction) of the factor analytic measurement theories, making it a versatile yet rigorous approach to theory development that offers avenues for achieving theoretical integration (e.g., by examining the relevance of dimensions posited by social identity theory of leadership in the context of dimensions posited by other perspectives such as leadership categorization theory; Epitropaki \& Martin, 2014; Lord et al., 1984; Offerman et al., 1994). 
So far, we have discussed the utility of a cognitive modeling approach for testing different representational assumptions about leader categories. Another useful feature of the approach is that a model-based analysis permits measurement of the necessity and relative importance of different attribute dimensions across different contexts. Factor analytic theories can identify attribute dimensions that are central to concepts such as leadership, however, they are limited in that they have no in-built mechanism to explain how the relevance of those dimensions might be modulated by contextual factors (e.g., the attributes of the specific candidates under consideration or the specificity of the leadership role in question). This issue is an important one, because researchers have observed that judgments of leaders and the attributes informing these judgments can vary as a function of whether leaders are judged in very general terms (e.g., "would this person be a good leader?”; Epitropaki et al., 2013; Junker \& van Dick, 2014) or in highly specific ones (e.g., "would this person be an effective US president?”; Pillai, Williams, Lowe, \& Jung, 2003; Simonton, 1994).

Categorization models address the issue of context-sensitivity by attaching attention weights to different attribute dimensions (Nosofsky, 1986). Such weights reflect the possibility that not all attribute dimensions are equally relevant for making a decision, allowing some attribute to influence the judgment process more strongly than others, and for the relative influence of each attribute to vary across contexts. Attention weights are assumed to affect the way similarity is determined within the model, accentuating attribute differences on attended dimensions while minimizing those on unattended dimensions. The effects of varying attention weights can be understood geometrically as the stretching of attended dimensions and the shrinking of unattended ones, as illustrated in the two bottom panels of Figure 1. The bottom left panel depicts an individual, $X$, in relation to effective and ineffective leader prototypes, $A$ and $B$, respectively. When attention is allocated equally 
across the two dimensions (i.e., advancing the interests of the in-group and embodying the ingroup), these attributes are equally influential in determining the proximity of $X$ to the two prototypes (i.e., how similar $X$ is to $A$ and $B$ ). Here, $X$ is most similar to the ineffective leader prototype, $B$. The bottom right panel shows a different allocation of attention that might arise from a change in context. In this scenario, attention is unevenly allocated across the two attribute dimensions, causing the similarity relations to change. Here, attention is heavily loaded on the degree to which the candidate embodies the in-group (horizontal dimension), and weakly loaded on the degree to which the candidate advances the interests of the ingroup (vertical dimension). Under this regime, $X$ is perceived as most similar to the effective leader prototype, $A$, despite no changes in any of the attribute values.

As sketched above, the cognitive modeling approach allows us to examine the generality of dimensional structures for leadership proposed by frameworks such as social identity theory of leadership. By examining patterns of attention weights across different attribute dimensions for making leadership judgments in different contexts, we can gain an understanding of the relative importance of these attribute dimensions and the degree to which they are sensitive to contextual factors. Further, the approach invites the possibility of examining when social identity-related information — or other attribute information — might be made more or less salient with regards to leadership judgments made in different contexts, enabling a more nuanced understanding of the multifaceted nature of leadership judgments.

\section{Theoretical and Empirical Goals of the Current Study}

In this article, we argue that the psychological process assumptions implied by theories of judged leader effectiveness (and leadership more generally) can be rigorously tested by incorporating cognitive modeling techniques that have been developed over the last 
several decades in the cognitive psychology literature. Working within the framework of social identity theory of leadership, we show how competitive model fitting can be used to assess the adequacy of different assumptions about category representations, and further show how assumptions about contextual modulation can be tested through inspection of model parameters. Our work is intended to serve as an illustrative example of how cognitive modeling can be used to rigorously test and develop theories of leadership in a way that connects core theoretical commitments about psychological processing (e.g., that leadership judgments can be viewed as the outcome of a categorization process) with relevant behavioral data (i.e., judgments of leader effectiveness).

Specifically, we use cognitive modeling to analyze leadership judgment data based on psychological dimensions outlined by social identity theorizing of leadership (Haslam et al., 2011; Steffens et al., 2014; van Dick et al., 2018). We contrast the fits of models incorporating prototype- and exemplar-based representations of leader categories, and show that an exemplar-based representation provides a highly accurate account of our data, whereas a prototype representation qualitatively fails to fit the data. The failure of the prototype model is noteworthy, as it highlights constraints in the way leadership prototypes ought to be conceptualized. The exemplar model accounts for approximately $90 \%$ of the variance in leadership judgments, and the results from this model reveal that some, but not all, of the underlying psychological dimensions of identity leadership contribute to people's judgments of leader effectiveness. Interpretation of the corresponding parameters of the prototype model leads to similar general conclusions. Our analysis provides a novel quantitative test of identity leadership theorizing, illustrating both advantages and limitations of this approach. More generally, our modeling results serve as an existence proof that judgments of leadership effectiveness can be interpreted as the outcome of an exemplar-based 
categorization process, and that this interpretation provides a better account of data than a process mediated by one form of prototype representation.

\section{Overview of the Model-Based Approach}

In this article, we integrate the social identity theory of leadership with cognitive models of categorization to directly compare different representational assumptions by fitting prototype (Minda \& Smith, 2011; Nosofsky, 1992a; Reed, 1972) and exemplar-based models to data (Nosofsky, 1986, 2011). According to these models, categorization decisions relevant to leadership (e.g., "is this person an effective leader?") are based on the similarity between a candidate leader and a cognitive representation of either a leader prototype or a collection of leader exemplars stored in memory. The more similar the candidate is to the effective leader representation, the more likely they will be classified as an effective leader. An important feature of these models is that they incorporate tuneable attention weights that reflect how influential different psychological dimensions are for making category decisions in different contexts - the higher the attention weight on a particular dimension, the more influential it is.

In addition to identifying the extent to which each dimension contributes to leadership judgments, a model-based approach permits analysis of the memory and response processes involved in making leadership judgments in different contexts. By analyzing how similarity is determined within models, we can gain further insight into how influential different sources of information in memory are for making judgments in different contexts. The exemplar model in particular can be used to understand the degree to which information is pooled across multiple exemplars to make a decision as opposed to being driven by a smaller number of specific exemplars (e.g., Nosofsky \& Zaki, 1998). In this way, our model-based analysis allows us to illustrate how memory and attentional processes transform the 
conceptual structure of identity leadership into judgments of a leader's effectiveness. The models provide further insight into factors that might influence people's judgments in different decision contexts (e.g., the propensity to judge an individual as an effective leader may depend on the specificity of the leadership role, potentially reflecting base rate information in the environment: a randomly selected individual is perhaps unlikely to be an effective national leader, but they may be an effective leader within their workplace).

In traditional applications of the exemplar and prototype models—often involving simple perceptual stimuli - the dimensional structure of the stimulus space is ascertained via multidimensional scaling (e.g., Nosofsky, 1992b; for a recent example in a natural category domain —igneous, metamorphic, and sedimentary rocks—-see Nosofsky, Sanders, Meagher, \& Douglas, 2018). In the current work, we assume the dimensional structure described by Haslam et al. (2011) and assessed and validated by Steffens et al. (2014) and van Dick et al. (2018). That is, we assume that all stimuli, prototypes, and exemplars are represented along psychological dimensions that correspond to the identity leadership dimensions of embodying, advancing, creating, and embedding a shared sense of 'we' and 'us'. The positioning of stimuli, prototypes, and exemplars along these leadership dimensions corresponds to the individual's normalized attribute ratings (i.e., we linearly transform ratings from a 7-point Likert scale to the interval $[0,1])$.

Given the prominence of prototype representation in the leadership literature (e.g., Haslam et al., 2011; Lord et al., 1984; van Knippenberg, 2011), it is reasonable to ask why we also consider an exemplar-based alternative. Our answer is twofold. First, the identity leadership literature to date does not provide formal theory or empirical tests of whether category representations of effective (identity) leaders reflect a summary representation of 
stimuli in the category (i.e., prototype structures) or to particular instances or exemplars of the category (i.e., exemplar structures; see McGarty, 1999, for extended discussion). It is therefore prudent to evaluate and compare the adequacy of both accounts. Second, we consider exemplar-based representation in particular due to the consistent success of exemplar models in the cognitive categorization literature (e.g., Nosofsky, 2000; Nosofsky \& Zaki, 2002; Rehder \& Hoffman, 2005; Zaki, Nosofsky, Stanton, \& Cohen, 2003). We stress that although our study contrasts the fits of exemplar and prototype models, the two forms of category representation can be viewed as end-points along a single theoretical continuum of cognitive abstraction. Exemplar-based representation is at the pole of no abstraction of information across items held in memory. There is a separate memory trace that corresponds to each individual exemplar containing leadership-relevant information. Encounters with stimuli create new exemplar memory traces, as information about these stimuli is retained in memory. Under exemplar-based representation, a voter might endorse a candidate if they are deemed sufficiently similar to other specific leaders the voter remembers as effective. Prototype representation is at the pole of maximal abstraction (i.e., a category is summarized by a single representation — often an average — containing leadership information that is abstracted across all category exemplars). In its strongest form, prototype representation only ascribes a memory trace to the prototype itself. Individual exemplar information is not retained, though the prototype can be updated by encounters with new exemplars. Under this form of prototype representation, a voter might endorse a candidate if they are deemed sufficiently similar to a summary representation of an average of effective leaders the voter remembers.

In the current article, we contrast the strong versions of exemplar and prototype representation - partly in keeping with common practice in the cognitive modeling literature, 
and partly due to theoretical and practical considerations that we elaborate on in the Discussion. However, we strongly suspect that the truth lies somewhere between, and note that other successful models such as the rational model (Anderson, 1991), the variable abstraction model (Vanpaemel \& Storms, 2008), and SUSTAIN (Love, Medin, \& Gureckis, 2004) adopt intermediate representational assumptions that are neither pure prototype nor pure exemplar representations (i.e., categories are represented by a number of clusters that summarize subsets of exemplars that share similar properties to one another). An emphasis on cluster-based representation, which can be viewed as a more fine-grained form of prototype representation in that different subsets of exemplars are described by their own summary representations, is perhaps more in line with Rosch's (1975; 1978; Rosch et al., 1976) theorizing about prototype representation, and has parallels in the implicit leadership theories literature (e.g., Lord et al., 1984). We discuss some of the challenges associated with developing these ideas in ways that are both theoretically justified and also amenable to formal evaluation in the Discussion. Our assessment of the prototype model and its attendant ramifications for categorization-based theories of leadership applies to a strong interpretation of prototype abstraction - though multiple-prototype models could be developed and tested in the same manner. It follows that we do not seek to make claims about whether all types of leadership judgments are based on either similarity to some form of prototype representation or an exemplar-based representation. Rather, our aim is to show how different theoretical assumptions can be articulated as formal mathematical models and evaluated quantitatively by fitting the models to data. More broadly, we believe our comparison of exemplar and prototype models serves an illustrative purpose of how leadership theorizing can profitably be combined with cognitive modeling to develop strong quantitative tests of representational assumptions. 


\section{The Prototype Model}

According to prototype theories (e.g., Posner \& Keele, 1968; Reed, 1972; Rosch, 1975, 1978), categorization decisions are based on the similarity between a stimulus and different category prototypes in memory. Category prototypes are represented as points in a multidimensional psychological space, reflecting average feature values possessed by category members (Nosofsky, 1992a; Minda \& Smith, 2011; Reed, 1972). Under the prototype model, the degree to which one believes a stimulus (e.g., the candidate being evaluated) will belong to a certain category (e.g., effective leaders) is based on how similar the stimulus is to the target category prototype (i.e., effective leaders), relative to the contrast category prototype (i.e., ineffective leaders). The higher the similarity between the stimulus and a given prototype, the more likely it is that the stimulus will be judged as belonging to the same category as that prototype. Formally, similarity between a stimulus $i$ and a prototype $P$ denoted $s_{\mathrm{iP}}$, is an exponential transformation of the distance between them,

$$
S_{i P}=e^{-c \cdot d_{i P}}
$$

where $d_{\mathrm{iP}}$ describes the distance between $i$ and $P$, and $c$ is an exponential decay constant that describes the steepness of the prototype similarity gradient (for theoretical discussion of these generalization gradients, see Shepard, 1987; Tenenbaum \& Griffiths, 2001). As values of $c$ increase, the similarity gradient becomes sharper, meaning that similarity to the prototype reduces at a faster rate.

The distance between stimulus $i$ and prototype $P$ is a weighted sum of the inter-item differences along each dimension in psychological space. Critically, different dimensions can be assigned greater or lesser weightings that reflect the relative importance of a dimension for making a particular judgment. For example, a voter might place lesser importance on whether a candidate embodies the ideals of the in-group and greater importance on whether a 
candidate is advancing shared in-group interests. Such weightings affect the proximity of the candidate to different prototypes, as illustrated in Figure 1. For psychologically separable dimensions (Garner, 1974) — which we assume here — distance is described as

$$
d_{i P}=\sum_{m=1}^{M} w_{m}\left|x_{i m}-x_{P m}\right|
$$

where $m$ indexes the different dimensions, and $x_{\mathrm{im}}$ defines the feature value of item $i$ along dimension $m$. The weight assigned to dimension $m$ is given by $w_{\mathrm{m}}$. Following Nosofsky (1986), we refer to $w_{\mathrm{m}}$ as attention weights and constrain them to sum to one across dimensions, reflecting capacity limitations. Referring back to the example of the voter, the distance between the candidate being considered and the prototypical candidate from a particular category (e.g., the prototypical effective leader) would equate to the sum of the differences between the candidate and prototype on each dimension of identity leadership, weighted by the importance of each of those dimensions. This means that more important dimensions have a stronger influence on whether the candidate is perceived as similar or dissimilar to the prototype.

Equation 2 yields a so-called city-block distance metric. We also modeled the data using the integral dimension form of Equation 2 that produces a Euclidean distance metric (see Nosofsky, 1986; 2011), where distance is calculated as the square root of the (weighted) squared distances along each dimension. The choice of distance metric had no substantial impact on either fits or patterns of parameter estimates - in almost all cases, the estimated values were nearly identical, resulting in identical theoretical conclusions. A summary of parameter estimates for models fitted with the Euclidean distance metric is presented in the Appendix. 
The probability with which stimulus $i$ is assigned to Category $J$ is given by an extension of Luce's (1959) choice rule,

$$
P(J \mid i)=\frac{b_{J} s_{i P_{J}}}{\sum_{K=1}^{K_{N}} b_{K} s_{i P_{K}}}
$$

The choice equation states that categorization of stimulus $i$ is determined by the similarity of $i$ to the Category $J$ prototype, divided by the total similarity of $i$ to each category prototype. The $b$ parameters denote response biases favoring a particular category, and are constrained to sum to one. When the $b$ parameters are equal across categories, no bias exists. When $b$ is unequal across categories, individuals are more likely to categorize stimuli as belonging to the category with the higher value of $b$, regardless of the similarity between the stimuli and that category's prototype. For example, an optimistic voter may be inclined to judge a candidate as an effective leader even though the candidate is not especially similar to the effective leader prototype. For simplicity, in the current study, we interpret the output of Equation 3 as being linearly related to the degree to which one judges an individual as a competent leader (a more generic evaluation of leadership aptitude with no specific designated role) or as an effective US President (a specific evaluation of leadership in a unique specific role). We choose these roles in particular as the former is without any specific detail about the nature of the role as a leader, whereas the latter is a highly specific role that is nevertheless highly familiar to members of the public, reflecting the varying scope of leadership roles considered in the literature (e.g., Dinh, Lord, Gardner, Meuser, Liden, \& Hu, 2014; Epitropaki et al., 2013; Junker \& van Dick, 2014; Pillai, Williams, Lowe, \& Jung, 2003; Simonton, 1994).

\section{The Generalized Context Model: An Exemplar-Based Model}


The exemplar model we consider is the Generalized Context Model (GCM; Nosofsky, 1986, 2011). Like the prototype model, categorization decisions under the GCM are based on similarity, not to category prototypes, but to labeled exemplars in memory. Category exemplars are represented as points in a multidimensional psychological space, and in this application, summarize information about known individuals (e.g., Barack Obama) along various attribute dimensions that is held in memory. In the case of the GCM, the degree to which one believes an individual is a member of the category of effective leaders is based on the relative summed similarity between the individual and all members of the effective leader category compared to all members of the contrast category. According to the exemplar-based view, a voter judges candidates based on their similarity to stored examples of candidates who they regard (and do not regard) as effective, rather than summary representations of those groups.

Similarity in the GCM is defined analogously to how it is defined in the prototype model, such that similarity between a stimulus $i$ and an exemplar $j$ denoted $s_{\mathrm{ij}}$, is given by,

$$
s_{i j}=e^{-c \cdot d_{i j}}
$$

where $d_{\mathrm{ij}}$ describes the distance between $i$ and $j$. Once again, $c$ is an exponential decay constant that determines the steepness of the similarity gradient of stored exemplars. Because multiple exemplars contribute to each decision, the $c$ parameter determines whether information is pooled across many or fewer exemplars. Higher values of $c$ produce sharper similarity gradients, such that decisions are driven primarily by the single most similar exemplar in memory (e.g., a voter basing their judgment on one or two politicians that are highly similar to the candidate being considered). At lower values of $c$, the similarity gradients are relatively shallow allowing multiple exemplars to influence a decision about a 
stimulus (e.g., a voter comparing a candidate to a larger number of politicians that vary in their similarity to the candidate in question).

The distance between stimulus $i$ and exemplar $j$ in psychological space is computed analogously to Equation 2,

$$
d_{i j}=\sum_{m=1}^{M} w_{m}\left|x_{i m}-x_{j m}\right|
$$

where $m$ again indexes the different dimensions, and the weight assigned to dimension $m$ is given by $w_{\mathrm{m}}$.

Because category assignment is based on summed similarity across exemplars in each category, the response rule for the GCM differs from that of the prototype model, though it retains the same general properties. In typical applications of the GCM, stimuli are probabilistically assigned to categories. The probability with which stimulus $i$ is assigned to Category $J$ is given by,

$$
P(J \mid i)=\frac{b_{J}\left[\sum_{j=1}^{n} s_{i j}\right]^{\gamma}}{\sum_{K=1}^{K_{N}} b_{K}\left[\sum_{k=1}^{n} s_{i k}\right]^{\gamma}}
$$

where the $b$ parameters again denote response biases favoring a particular category response. The GCM response rule also includes a response scaling parameter, $\gamma$ that controls how deterministically the model responds. This parameter is constrained such that $\gamma \geq 1$. When $\gamma=$ 1, category assignment is proportional to the relative similarity of the stimulus to exemplars of different categories. As $\gamma$ increases, category assignment becomes more deterministic, favoring the category whose exemplars are most similar to the stimulus. The response scaling parameter is not present in the prototype model's response rule, as it is not mathematically identifiable given how similarity is computed in Equation 1. Consequently, the $c$ parameter in the prototype model reflects the combined effects of the similarity gradient as well as 
response scaling (Nosofsky \& Zaki, 2002). The interpretation of Equation 6 follows that of Equation 3. Ignoring response bias, categorization of stimulus $i$ is determined by the summed similarity of $i$ to exemplars belonging to Category $J$, divided by the summed similarity of $i$ to exemplars belonging to Category $J$, plus the summed similarity of $i$ to exemplars belonging to each other category. This means that a voter's decision about a candidate will be based on the summed similarity of the candidate to all stored examples of effective leaders, versus the candidate's summed similarity to all stored examples of ineffective leaders.

\section{Pilot Study: Identifying Recognizable Individuals}

To get a clearer sense of how judgments of leadership ability apply to actual individuals — rather than verbal descriptions of hypothetical people—we sought to construct a stimulus set comprising highly recognizable public US figures. Because we had planned on targeting American participants in the main study, the pool of public figures we considered was focused primarily on American culture and society. Example lists from which identities were sourced include Reader's Digest's list of the 100 Most Trusted People in America, and the Smithsonian Magazine's list of the 100 Most Significant Americans of All Time. This pool of identities included several fictional characters. We decided not to exclude these fictional identities from the pool, as some of them can be viewed as idealized representations of cultural values (e.g., superheroes, such as Superman and Wonder Woman), or as depictions of a certain cross-section of members of American society (e.g., Fred Flintstone as an embodiment of a member of the working-class). We were therefore curious to see how these characters were rated in terms of leadership abilities - their ontological status notwithstanding. An analysis of the data excluding fictional and historical figures is reported in the Appendix, and yielded the same general theoretical conclusions. To ensure that the stimuli were well-known to participants, we conducted an initial familiarity study designed to 
identify highly recognizable identities in the US. We began with a pool of 1147 identities compiled from various lists of notable figures from American society to ensure that we had a mix of modern and historical figures from a number of different walks of life (e.g., athletes, politicians, entertainers, academics, and business people), including some fictional characters (e.g., Superman). We then obtained images for each of the stimuli from the web, where they were front-facing and bearing a neutral expression. If available, photographs were used to visually portray the individuals, otherwise portraits were used (e.g., to depict historical figures). For this pilot study, we recruited 545 participants via Prolific Academic, each of whom provided familiarity ratings for a randomly selected subset of 80 stimuli from the pool. This ensured approximately 50 familiarity ratings for each stimulus in the pool. For each stimulus, participants were shown their name, an image, and were prompted to rate the extent they agree with the statement: "I am familiar with [Stimulus]," on a 7-point Likert scale $(1=$ Strongly Disagree; 7 = Strongly Agree). We then generated an average familiarity score for each stimulus by averaging across available familiarity ratings. The final stimulus set that we then used in the experiment reported below comprised 80 stimuli randomly selected from the 100 most highly recognized stimuli along with their associated images. We henceforth use the term stimuli to refer to the 80 stimulus identities used in the study described below.

\section{Method}

\section{Participants}

We recruited a total of 965 participants who resided in the US via Prolific Academic. Participants were remunerated $£ 1.00$ (around US\$1.40 at the time) in exchange for completing a survey taking approximately 10 minutes. We excluded data from a total of 63 participants, 42 of whom failed to complete the survey, and another 21 of whom failed an 
attention check question, "This is a control question please select not at all (1)”, leaving a total of 902 participants whose data entered the analysis. Analyses including the data of the 21 participants who failed the attention check are reported in the appendix - there are no meaningful differences in the results or conclusions. Of these participants who provided demographic data, 442 identified as male, 448 as female, six as transgender, five as other and one participant preferred not to say. Participants ranged in age from 18 to 82 years $(M=$ $34.57 ; S D=12.71)$.

Table 1. Survey items organized by dimension for items adapted from the identity leadership inventory (Steffens et al., 2014), and by individual attribute for authenticity, identification with the individual, overall leader competence, and judged effectiveness as a US president.

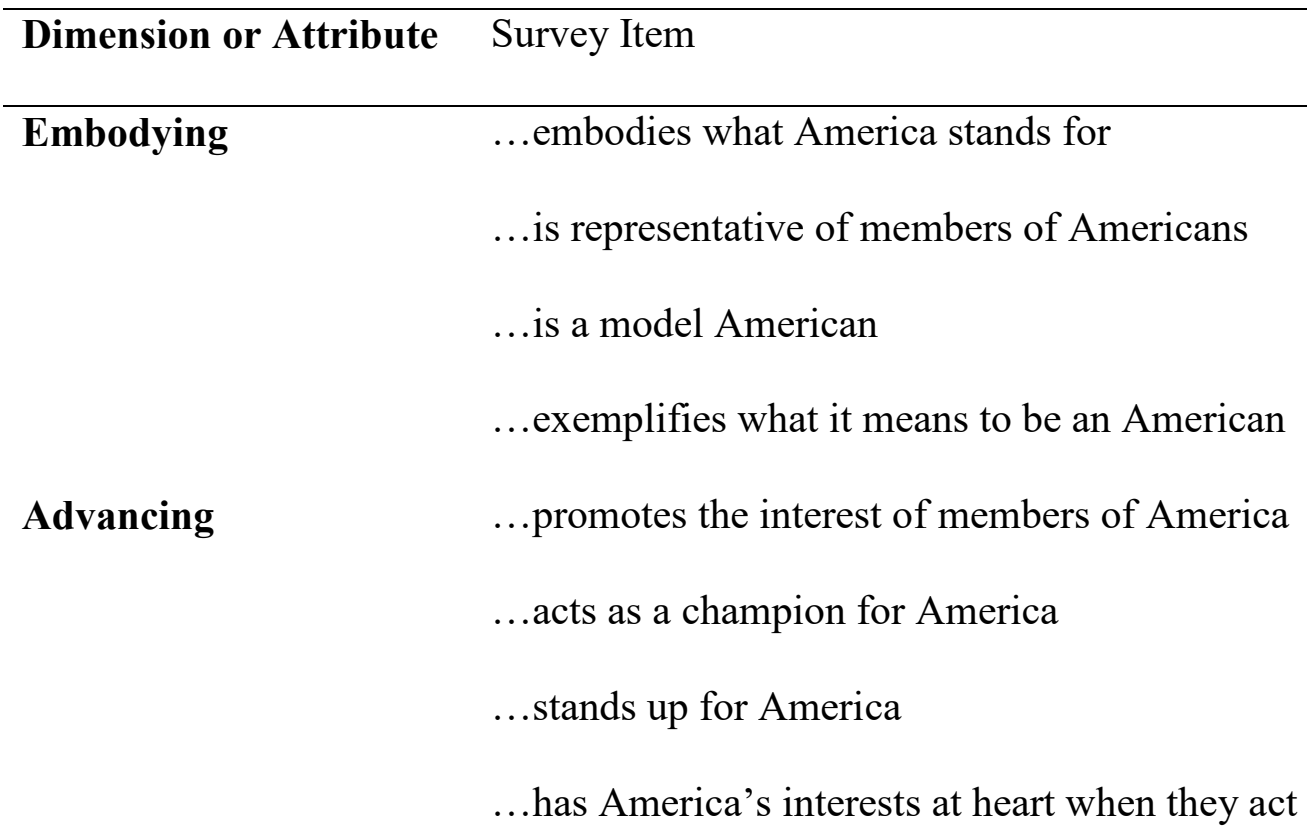

Creating

...makes people feel as if they are part of the same group

...creates a sense of cohesion within America

...develops an understanding of what it means to be an

American

....shapes Americans' perceptions of US values and ideals

Embedding $\quad$...devises activities that bring Americans together 
...arranges events that help America function effectively

...creates structures that are useful for America

Authenticity This person is authentic

Personal Identification I identify with this person

Leader Competence This person is a competent leader

Effective US President This person would make an effective US president

Table 2. Demographic questions presented after all stimuli were rated. Rating questions had the same anchor values and labels as the stimulus ratings (i.e., $1=$ Not at All; $7=$ Completely). The attention check question is italicized in the table. Questions about political beliefs were rated on a 7-point Likert scale with $1=$ Left $/$ Liberal; $4=$ Neutral; and $7=$ Right/Conservative.

Please rate the extent to which these statements apply to you:

I identify with America

I identify with Republicans

This is a control question please select not at all (1)

I identify with Democrats

In which year were you born?

What is your gender?

What is your nationality?

What are your political beliefs from left to right wing on:

Social Issues (e.g., immigration, abortion)

Economic Issues (e.g., social welfare, tax cuts)

What is your Prolific ID?

Do you have any comments about this study? 


\section{Procedure}

Participants accessed the survey through Prolific Academic. After reading a brief description of the study aims and the task instructions, participants were invited to indicate that they consented to participate by continuing onto the survey. Upon completing the survey, participants received a code that could be used to receive payment for participation. Each participant was presented with a series of nine randomly selected stimuli from the final pool of 80 stimuli. For each of the nine presented stimuli, participants indicated their responses to the 19 questions listed in Table 1. Despite each participant rating only a small subset of the total number of stimuli, the total number of participants recruited ensured that we were able to collect approximately 100 sets of ratings for each of the 80 stimuli (minimum $=96$; maximum $=106$ ). After providing attribute ratings for the nine presented stimuli, participants provided responses to some demographic questions listed in Table 2. 


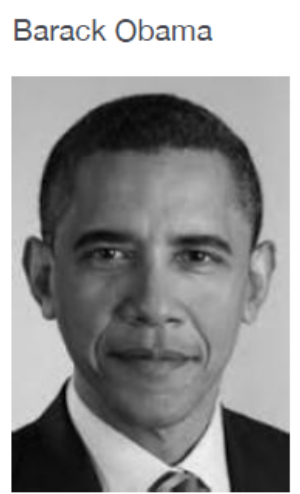

Barack Obama

\begin{tabular}{|c|c|c|c|c|c|c|c|}
\hline & Not at al & & & & & & mpletely \\
\hline & 1 & 2 & 3 & 4 & 5 & 6 & 7 \\
\hline $\begin{array}{l}\text {... embodies what } \\
\text { America stands for }\end{array}$ & O & $\mathrm{O}$ & $\mathrm{O}$ & $\mathrm{O}$ & O & O & $\mathrm{O}$ \\
\hline $\begin{array}{l}\ldots \text { is representative of } \\
\text { members of Americans }\end{array}$ & O & $\mathrm{O}$ & $\mathrm{O}$ & $\mathrm{O}$ & O & O & $\mathrm{O}$ \\
\hline ... is a model American & O & $\mathrm{O}$ & 0 & 0 & 0 & 0 & $\mathrm{O}$ \\
\hline $\begin{array}{l}\text {... exemplifies what it } \\
\text { means to be an } \\
\text { American }\end{array}$ & O & O & O & O & 0 & O & $\mathrm{O}$ \\
\hline $\begin{array}{l}\text {... promotes the } \\
\text { interests of members of } \\
\text { America }\end{array}$ & O & $\mathrm{O}$ & $\mathrm{O}$ & $\mathrm{O}$ & O & O & $\mathrm{O}$ \\
\hline $\begin{array}{l}\text {... acts as a champion } \\
\text { for America }\end{array}$ & O & $\mathrm{O}$ & $\mathrm{O}$ & $\mathrm{O}$ & 0 & 0 & $\mathrm{O}$ \\
\hline $\begin{array}{l}\text {... stands up for } \\
\text { America }\end{array}$ & O & $\mathrm{O}$ & $\mathrm{O}$ & $\mathrm{O}$ & $\mathrm{O}$ & O & $\mathrm{O}$ \\
\hline
\end{tabular}

Figure 2. Screenshot showing how stimuli and survey questions were presented. For each stimulus, participants indicated the degree to which they believed the individual embodied various leadership attributes.

In the survey (programmed on Qualtrics), participants were presented with a stimulus (e.g., the name Barack Obama along with an image), before indicating the degree to which they agreed with several item statements on a 7-point Likert scale $(1=$ Not at all; $7=$ Completely; see Figure 2). More specifically, we used 15 item statements assessing the four dimensions of identity leadership (i.e., embodying, advancing, creating, embedding a shared collective identity) from Steffens et al. (2014). We further consider two other dimensions corresponding to authenticity and identification with the leader as additional variables to assess whether identity leadership dimensions contribute to leadership judgments when 
controlling for a leader's perceived authenticity (Gardner, Cogliser, Davis, \& Dickens., 2011; Neider \& Schriesheim, 2011) and the extent to which individuals identify with the leader at a personal level (Ashforth, Schinoff, \& Rogers, 2016; Sluss \& Ashforth, 2007). Authenticity was assessed via a single-item created for the purpose of the present experiment to tap into ideas proposed by Authentic Leadership work (Gardner et al., 2011). Personal identification with the individual was assessed via an adaptation of the single-item social identification scale from Postmes, Haslam, and Jans (2013) that referred to the candidate leader instead of the group as target. We included only single-item measures of authenticity and personal identification for practical reasons (i.e., to limit the number of ratings people needed to provide to avoid potential risk of task disengagement). While multiple-item measurements can yield more reliable estimates, we point out that single items can be useful and reliable (as discussed by Postmes et al., 2013), though future work would benefit from also using more extensive measurements. Notwithstanding recent critiques of some contemporary conceptualizations of leader attributes (such as authenticity; Alvesson \& Einola, 2019; Kaufman, 2019), we included these latter measures in an exploratory fashion, given the vast number of leadership theories (Dinh et al., 2014) and little consensus on the key attributes that make good leaders. Their inclusion in our research enables us to get an initial impression of these attributes in relation to other leadership constructs that feature in social categorization and identity leadership theories. Finally, we assessed participants' judgments of the individual's overall leader competence and whether the individual would make an effective US President. There are some differences between the attributes derived from social identity theorizing of leadership and those commonly used in other leader categorization theories (e.g., Lord et al., 1984). We do not wish to imply that attributes based on social identity theory of leadership are necessarily preferable to other attributes with regards to leadership evaluations. Rather, our motivation for focusing on attributes within a social 
identity theory framework serves an illustrative purpose to show how a formal cognitive modelling framework can be integrated with, and used to inform understanding of, an attribute structure of social identity theory of leadership. The modeling framework also allows for attributes from multiple theories to be considered simultaneously, creating an avenue for formally integrating various leadership theories. For example, although we have focused mainly on attributes derived from social identity theory of leadership, we also incorporated controls in the form of several other alternative attributes based on other theories.

Because each participant rated all attributes of each stimulus they were presented with, there are potential concerns regarding common-source bias (e.g., Avolio, Yammarino, \& Bass, 1991). For example, rating a stimulus favorably in one attribute may lead to similarly favorable ratings on other attributes, potentially introducing artificial correlations among variables. Nevertheless, this is difficult to avoid in the present work, and indeed, is a necessary feature in research that seeks to answer the kinds of questions we are interested in (for discussion of these issues, see Avoilio et al., 1991; Dionne, Yammarino, Atwater, \& James, 2002). Specifically, interpreting the GCM and prototype models as psychological theories of leadership judgments - in line with their application in the cognitive literaturerequires that the psychological representation of the stimuli along their attribute dimensions (i.e., attribute ratings) are intrapsychologically relatable to outcome judgments (i.e., ratings of leader competence and presidential effectiveness). This can only be accomplished by having the same people provide information about all attributes of a given stimulus. Methods of addressing common-source bias, such as having one set of participants provide attribute ratings and another provide outcome ratings would decouple this information, limiting the interpretation of the models as psychological theories. At the same time, our study limits the 
influence of common-method bias by incorporating a direct test of competing theoretical accounts (viz. fitting cognitive models to data), avoiding experimental characteristics that might lead participants to respond in a way that favors one account over another. We return to this issue in the discussion.

For subsequent modeling purposes ratings of overall leader competence were used to determine the degree to which an individual was categorized as being a competent leader. The complete list of survey items is provided in Tables 1 and 2.

\section{Results}

We begin with a summary of descriptive data. Raw data and processed data for our main analyses have been posted on the Open Science Framework at https://osf.io/4h2t3/. As this is a large data set, we focus on presenting graphical summaries of the rating data before providing a detailed discussion of the modeling results. We present a brief summary of preliminary data screening based on questionnaire finishing times and response patterns in the Appendix. Figure 3 presents histograms showing the distributions of mean ratings for the 80 stimuli along the four dimensions of identity leadership, authenticity, personal identification with the target stimulus, as well as the focal dependent variables of overall leader competence and perceived effectiveness as a US President. All of the measures show fairly symmetrical unimodal distributions, though authenticity, identification with the stimulus, and presidential effectiveness show modest skew. 
Table 3. Correlation matrix for all rated attribute dimensions averaged across the 80 stimulus identities. For items assessing the four dimensions of social identity theorizing of leadership, individual attribute ratings were averaged to yield a dimensional rating for each stimulus identity. Mean ratings (and standard deviations) are presented along the diagonal.

\begin{tabular}{|c|c|c|c|c|c|c|c|c|}
\hline & Embodying & Advancing & Creating & Embedding & Authenticity & Identification & Lead Comp & Pres Effect \\
\hline Embodying & $4.35(1.45)$ & & & & & & & \\
\hline Advancing & 0.84 & $4.28(1.51)$ & & & & & & \\
\hline Creating & 0.83 & 0.85 & $4.33(1.46)$ & & & & & \\
\hline Embedding & 0.69 & 0.77 & 0.79 & $3.91(1.57)$ & & & & \\
\hline Authenticity & 0.65 & 0.61 & 0.61 & 0.53 & $4.83(1.62)$ & & & \\
\hline Identification & 0.62 & 0.60 & 0.61 & 0.58 & 0.55 & $3.21(1.77)$ & & \\
\hline Lead Comp & 0.67 & 0.69 & 0.67 & 0.63 & 0.63 & 0.61 & $4.15(1.58)$ & \\
\hline Pres Effect & 0.58 & 0.61 & 0.57 & 0.58 & 0.49 & 0.63 & 0.69 & $3.26(1.67)$ \\
\hline
\end{tabular}


For each of the 80 stimuli, we also present mean ratings for the two focal dependent variables. Figure 4 shows how each stimulus rated in terms of general leader competence, whereas Figure 5 shows how each stimulus rated in terms of perceived effectiveness as a US President. We present histograms illustrating the distribution of ratings for each stimulus in the Appendix.

Table 4. Cronbach's $\alpha$ values for the four social identity dimensions that aggregated information across multiple questionnaire items. Mean values, averaged across stimuli, are reported along with the minimum and maximum values.

\begin{tabular}{|c|c|c|c|}
\hline Aggregate Dimension & Mean Cronbach's $\alpha$ & Minimum $\alpha$ & Maximum $\alpha$ \\
\hline Embodying & 0.93 & 0.88 & 0.98 \\
\hline Advancing & 0.95 & 0.90 & 0.99 \\
\hline Creating & 0.92 & 0.86 & 0.97 \\
\hline Embedding & 0.92 & 0.81 & 0.98 \\
\hline
\end{tabular}

Correlations among variables, as well as means and standard deviations, are presented in Table 3. To compute dimensional values for each stimulus, we averaged across participant responses assessing each individual social identity factor to construct the four dimensional ratings for that stimulus. For each stimulus, we then computed correlations between all attributes (i.e., the four identity leadership dimensions, authenticity, and identification with the stimulus, leader competence, and presidential effectiveness) as well as means and standard deviations, which were all calculated at the level of individual stimuli (i.e., aggregating data across participants who rated those particular stimuli). To compute the correlations shown in the Table, we averaged the correlation matrices (and associated $p$ values) across the 80 stimuli. We also computed Cronbach's $\alpha$ values for the four social 
identity dimensions that involved aggregating across multiple questionnaire items.

Cronbach's $\alpha$ wascalculated for each stimulus. The values averaged across stiuli, as well as the minimum and maximum values are shown in Table 4.
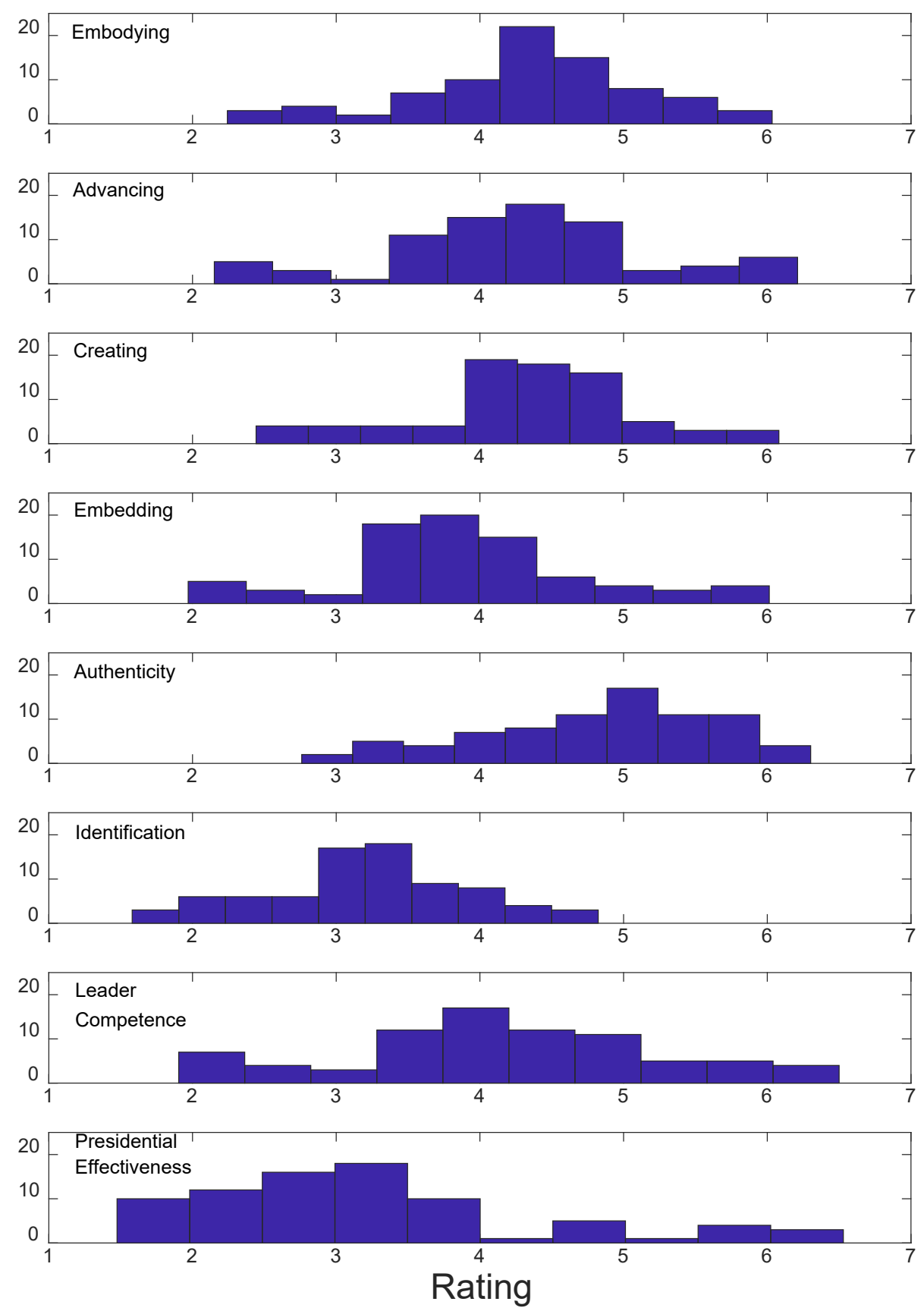

Figure 3. Histograms showing distributions of mean ratings for each of the 80 stimuli along each attribute dimension. 


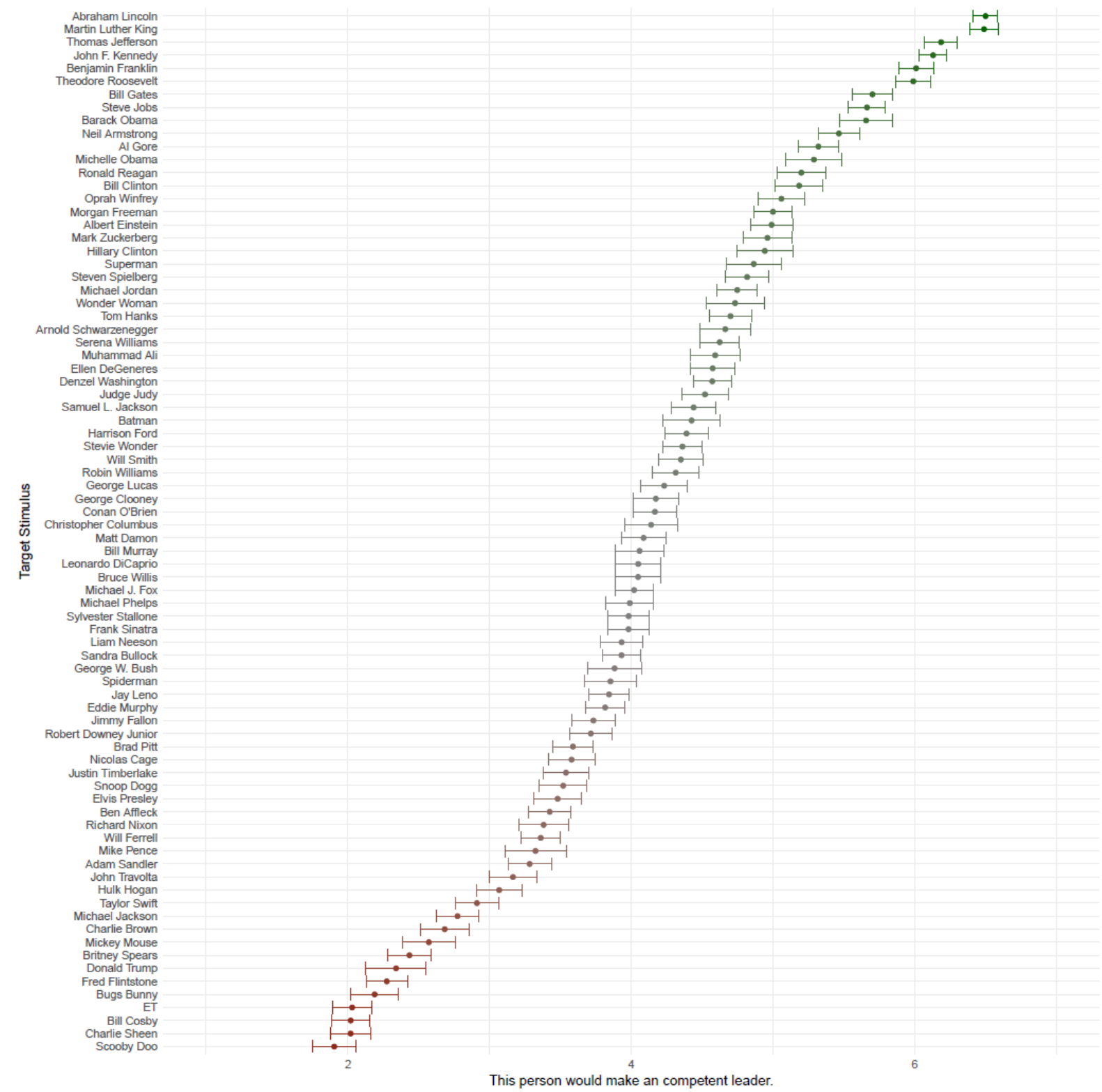

Figure 4. Mean leader competence ratings ( \pm 1 standard error of the mean, calculated for each stimulus by aggregating data across participants who rated that stimulus) for each of the 80 stimuli. Stimuli are ranked in the figure from highest-rated to lowest-rated. 


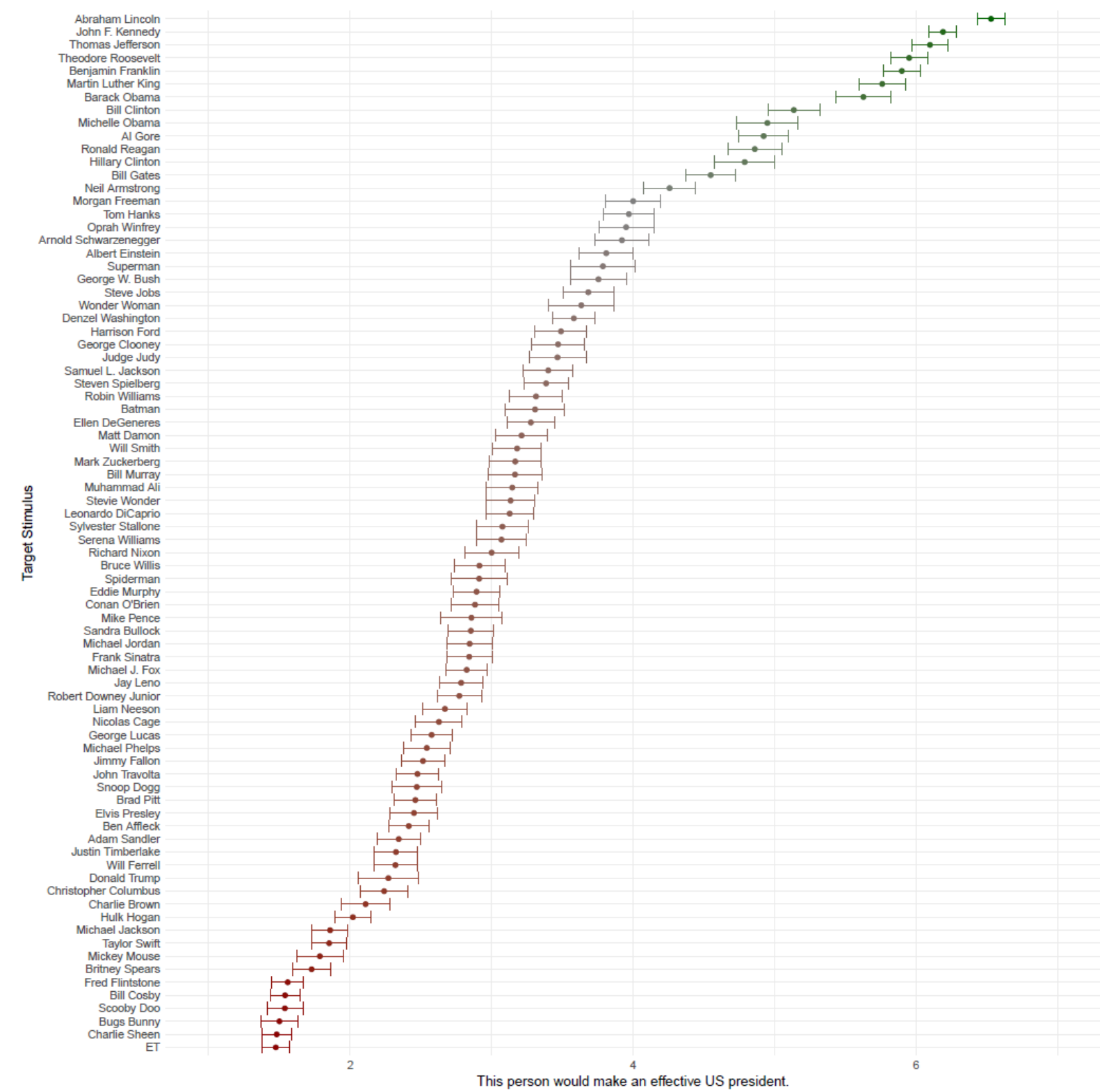

Figure 5. Mean presidential effectiveness ratings ( \pm 1 standard error of the mean, calculated for each stimulus by aggregating data across participants who rated that stimulus) for each of the 80 stimuli. Stimuli are ranked in the figure from highest-rated to lowest-rated.

\section{Computational Modeling}

\section{Specifying the Models: Continuous Category Membership}

We presented general overviews of the GCM and the prototype model in the introduction. Typically, the models are applied to data from categorization tasks, where 
people explicitly assign stimuli to categories based on learned experience with labeled training exemplars. In our study, however, we do not have an explicit training phase, where stimuli are attached to category labels. Instead, we assume that (1) people are familiar with the set of stimulus identities, which was confirmed by our pilot study, and (2) that the mean rating for overall leader competence reflects the degree to which a stimulus is viewed as belonging to the "Competent Leader" category. Because the leader competence rating is continuous, rather than binary, we interpret this as reflecting the probability with which identity is categorized as a competent leader by an "average" participant. This continuous measure of category membership requires some changes to how prototypes are constructed and how category similarity is computed in the GCM, as every stimulus in our study is considered a member of both the Competent Leader category as well as the Not Competent Leader category, albeit to differing degrees.

Regarding prototype construction, rather than reflecting a simple average of features - resulting in identical category prototypes, as the same individuals belong to each category - feature values are weighted by the degree to which an individual belongs to (say) the Competent Leader category. As a result, an identity that is assigned to the Competent Leader category $99 \%$ of the time will have a proportionately stronger influence on the Competent Leader prototype than an identity assigned to the Competent Leader category $1 \%$ of the time. Beyond this change in the way category prototypes are constructed, the model is otherwise identical to our earlier description.

Regarding the GCM, the influence of each exemplar, when calculating similarity to the Competent Leader category, is weighted by the mean competence rating for that exemplar. Calculating similarity to the Not Competent Leader category is weighted by 1 
minus the competence rating. Formally, this is accomplished by adding a term that weights the degree to which exemplar $j$ belongs to Category $J, v_{\mathrm{j} J}$, so the choice rule presented in Equation 6 becomes

$$
P(J \mid i)=\frac{b_{J}\left[\sum_{j=1}^{n} v_{j J} s_{i j}\right]^{\gamma}}{\sum_{K=1}^{K_{N}} b_{K}\left[\sum_{k=1}^{n} v_{k K} s_{i k}\right]^{\gamma}}
$$

Now, the relevant summations extend across all exemplars, as each stimulus identity is associated with both the "Competent Leader" and "Not Competent Leader" categories, albeit to differing degrees.

\section{Model Fitting Procedures}

Our main theoretical question was whether the prototype and exemplar models could successfully account for the leader competence ratings as well as the perceived effectiveness as a US president using the four identity leadership dimensions as the basis for these

judgments. To address our research question, we first fitted the models to the data in order to estimate their parameters. We then compared the fit of the two models in order to evaluate the adequacy of the theoretical assumptions instantiated by the two models. We assess this using both qualitative visual assessment of overall model fit as well as formal model selection, using the Akaike information criterion (AIC; Akaike, 1974). Finally, we assess the parameter estimates to gain insight into how different attributes were weighted in order to accurately predict the data. All analyses were conducted using Matlab (version 2017b).

We fit the models to the competence and presidential effectiveness ratings separately in order to analyze potential differences in memory processes, response biases, and the way identity leadership attributes were weighted between the two judgments. In each case, stimuli were represented along the embodying, advancing, creating, and embedding dimensions 
described by Haslam et al. 2011 (see also Steffens et al.; 2014; van Dick et al. 2018) as well as perceived authenticity and the degree to which individuals identify with the potential leader at a personal level. For both models, we freely estimated attention weight parameters for the six dimensions from the data, the similarity gradient parameter, $c$, as well as response bias favoring a positive rating, which we denote $b_{+}$. For the GCM, we also estimated the value of the response scaling parameter, $\gamma$. Because the attention weight parameters and the response bias parameters are each constrained to sum to one (see Minda \& Smith, 2011; Nosofsky, 1986; 2011), a total of seven free parameters were estimated from the data for the prototype model, and eight free parameters were estimated for the GCM. The modeling analyses each achieve a data reduction factor of around 10-to-1 (i.e., 79 data degrees of freedom accounted for by 7 or 8 freely estimated parameters). Parameter values were adjusted to minimize the summed squared error between data and model predictions (i.e., the output of Equations 3 and 7 as compared with the normalized rating data for either leader competence or presidential effectiveness, depending on the analysis) using Matlab's (version 2017b) implementation of the simplex algorithm (Nelder \& Mead, 1965). This algorithm takes an initial set of parameter values — we randomly generated starting values, using several different sets of starting values to allay concerns about local minima—and iteratively adjusts them in order to minimize some criterion (e.g., summed squared error, or a negative log likelihood). Parameter values are adjusted until no further reductions in the criterion are possible (see Farrell \& Lewandowsky, 2018, for a highly accessible tutorial introduction). We used a maximum likelihood approach to estimate model parameters, assuming a Gaussian error model, whereby we estimated model parameters to minimize the negative log likelihood of the model, defined as

$$
-\ln L=\frac{n}{2} \ln \left(\frac{S S E}{n}\right)
$$


where $n$ corresponds to the number of cell means we are fitting the model to, which is equal to the number of stimuli (i.e., $n=80$ ). SSE refers to the summed squared error between the data and the model predictions, and $l n$ denotes the natural logarithm.

When fitting the exemplar model, to avoid self-similarity effects, we used a leaveone-out method for generating model predictions, ensuring that information about a specific stimulus never contributed to the model's ability to account for its own competence or presidential effectiveness ratings. Because exemplars in our implementation of the model are probabilistically associated with both categories, having an exemplar corresponding to the stimulus in memory would artificially improve the fit of the model, and so our approach can be viewed as providing a more conservative test of the model. This approach to testing the exemplar model can be viewed psychologically as examining situations where the exemplar model does not "know" the identity of the current to-be-judged stimulus. Accordingly, for each of the 80 stimuli, we assumed that leader competence or perceived presidential effectiveness ratings were based on similarity to the 79 other stimuli in the data set. For the prototype model, because the representations are based on feature averages computed across all 80 stimuli, self-similarity effects were of lesser concern, and so we included information about all stimuli for determining category prototypes, regardless of which stimulus was being evaluated. Best fitting parameter estimates for all analyses as well as model fits are summarized in Table 5. 
Table 5. Model fit statistics and best-fitting parameter estimates for fits to the Leader Competence and Presidential Effectiveness data sets. Parameter estimates for attention weights and response bias are normalized.

\begin{tabular}{|c|c|c|c|c|c|c|c|c|c|c|c|}
\hline Data Set & $\ln L$ & $R^{2}$ & Embodying & Advancing & Creating & Embedding & Authenticity & Identification & $c$ & $\gamma$ & $b_{+}$ \\
\hline $\begin{array}{l}\text { Prototype: } \\
\text { Leader } \\
\text { Competence }\end{array}$ & 187.80 & 0.72 & 0.17 & 0.23 & 0.00 & 0.36 & 0.08 & 0.17 & 8.90 & - & 0.53 \\
\hline $\begin{array}{l}\text { Prototype: } \\
\text { US } \\
\text { Presidential } \\
\text { Effectiveness }\end{array}$ & 171.17 & 0.65 & 0.00 & 0.34 & 0.00 & 0.38 & 0.00 & 0.29 & 9.78 & - & 0.36 \\
\hline $\begin{array}{l}\text { GCM: } \\
\text { Leader } \\
\text { Competence }\end{array}$ & 221.20 & 0.88 & 0.00 & 0.33 & 0.00 & 0.47 & 0.11 & 0.09 & 7.84 & 2.64 & 0.47 \\
\hline $\begin{array}{l}\text { GCM: US } \\
\text { Presidential } \\
\text { Effectiveness }\end{array}$ & 223.51 & 0.91 & 0.00 & 0.51 & 0.00 & 0.45 & 0.00 & 0.05 & 40.97 & 1.34 & 0.33 \\
\hline
\end{tabular}

Note. $\ln \mathrm{L}=$ Log Likelihood; Identification = Identification with the target stimulus. 


\section{Analysis of Leader Competence Data}

In most applications of the prototype and exemplar models, category membership for stimuli is predicted on the basis of the known category membership of learned exemplars. Our first analysis is most closely related to this traditional application of the model in that we are attempting to predict leader competence ratings for each stimulus given category assignments based on competence ratings for the other 79 stimuli in the data set or the Competent Leader and Not Competent Leader prototypes. In order to interpret the parameter estimates psychologically, we need to first establish whether the models provide a good account of the data. We do this by visually summarizing fits to data by plotting the model predicted values against normalized average rating values. Figures 6 and 7 respectively show the correspondence between prototype and exemplar model predictions and data for the overall leader competence data. 


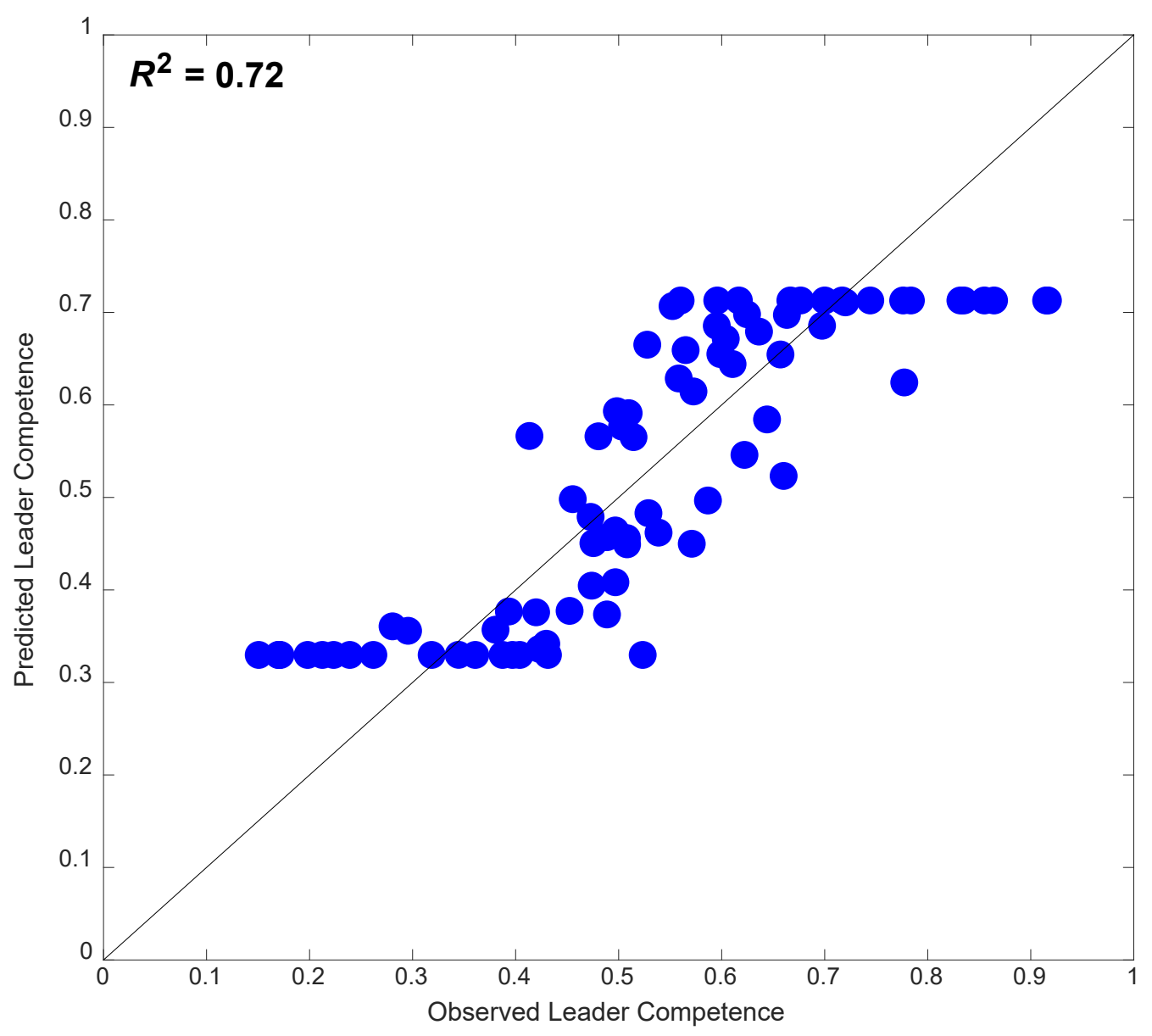

Figure 6. Observed leader competence ratings plotted against predicted values of the prototype model. Each data point in the plot corresponds to one of the 80 stimuli.

For the prototype model, the correspondence between theoretical predictions and data is not unreasonable, with $R^{2}=0.72$. Inspection of Figure 6 , however, reveals some critical shortcomings of the model. Most notably, the model is unable to predict the full range of competence ratings, as Equation 3 assigns stimuli to categories in direct proportion to their relative similarity to the two prototypes. In our data set, the separation of the prototypes in psychological space is limited, and consequently there is an upper limit on the relative similarity between a stimulus and the two prototypes, truncating the range of ratings that the 
model can predict. Increasing the value of the $c$ parameter could increase the range of predicted values, but evidently, this would come at the expense of correctly predicting the majority of ratings, which fall around the middle range of the scale. For these reasons, we conclude that our standard implementation of the prototype model does not provide an acceptable account of the data.

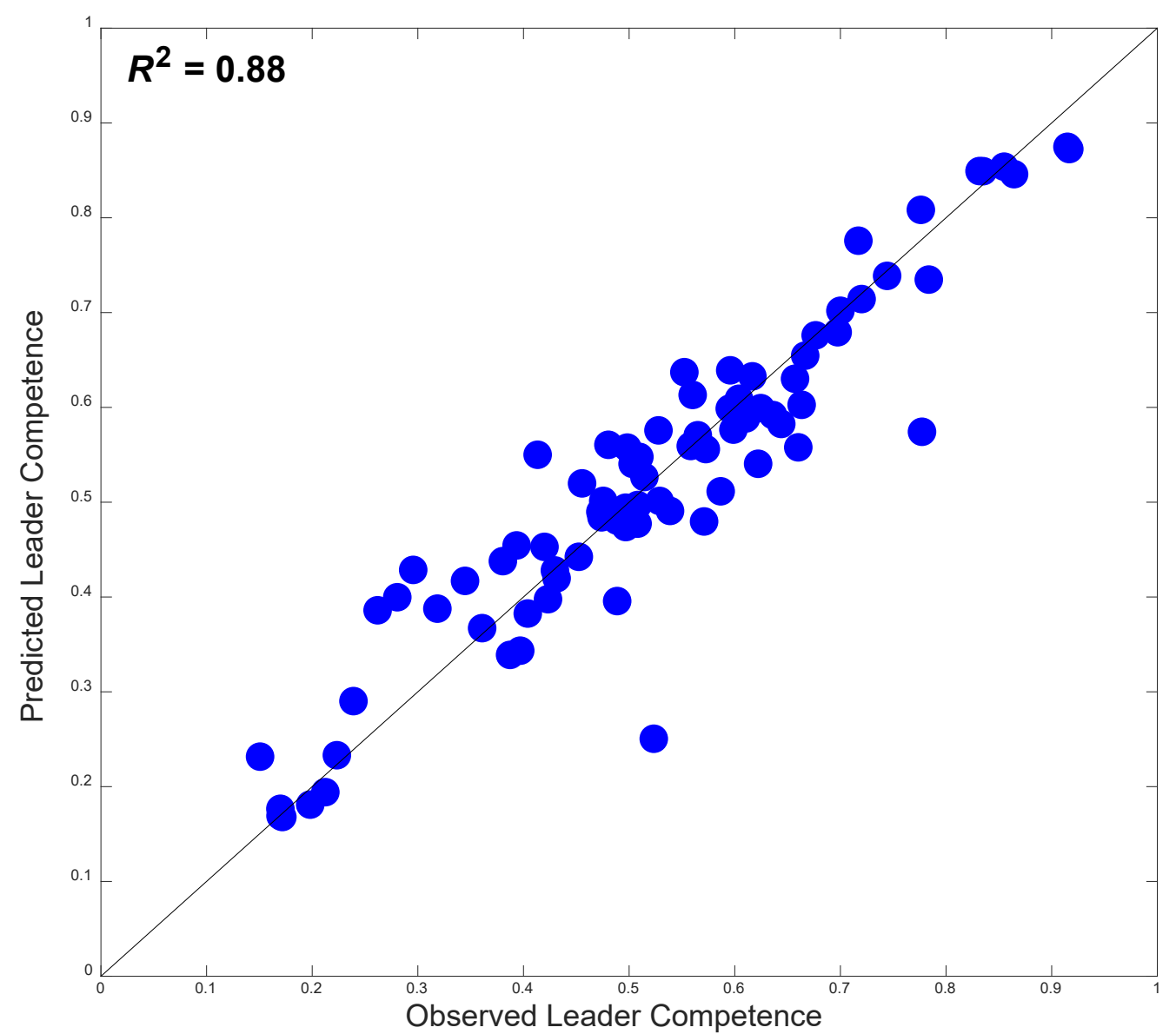

Figure 7. Observed leader competence ratings plotted against GCM-predicted values. Each data point in the plot corresponds to one of the 80 stimuli.

For the GCM, the correspondence between theoretical predictions and data is much better, with $R^{2}=0.88$. The quality of fit is in line with previous applications of the model to 
perceptual category learning data (e.g., Nosofsky, 1986, 2011). There is no systematic evidence of misfit, as leader competence is accurately captured by the GCM across the entire range of rating values, including the more extreme ends of the rating scale (see Figure 7). Formally, we can compare the fit of the GCM to that of the prototype model via the Akaike information criterion (AIC), which is defined as

$$
A I C=-2 \ln L+2 k
$$

The first term is twice the negative log likelihood of the model, from Equation 8, and $k$ refers to the number of free parameters in the model. For the prototype model, $k=7$. For the GCM, $k=8$. The AIC provides a way of balancing goodness of fit-summarized by the model likelihood - against model complexity. Models with lower relative AIC are preferenced over those with higher relative AIC. Based on AIC, the GCM can be formally preferenced over the prototype model, as it has a lower AIC value (i.e., -426.40 for the GCM vs. -361.60 for the prototype model).

The good fit of the GCM to the data provides in-principle support for the idea that leader competence ratings can be viewed as the outcome of an exemplar-based categorization process. While model fit alone cannot ever be held as definitive "proof" that the psychological processes instantiated by the model are responsible for generating the data, successfully accounting for the data is a critical demonstration of the viability of the process. Keeping these considerations in mind, we now provide a psychological interpretation of the parameters estimates of the model.

We first consider how the four dimensions of identity leadership contribute to ratings of overall leader competence. Analysis of the best-fitting attention weights in Table 5 shows, somewhat surprisingly, that only the advancing and embedding dimensions produce strong 
contributions toward predicting leader competence, producing normalized attention weights of 0.33 and 0.47 , respectively. The attention weights attached to the embodying and creating dimensions were both equal to zero, showing that these dimensions were entirely irrelevant (or unnecessary) for predicting leader competence ratings. Notably, leader authenticity and personal identification with the leader each produced attention weights of approximately 0.10 , indicating modest contributions toward predicting leader competence ratings. Competence ratings showed little evidence of having systematically higher or lower values, as suggested visually by the horizontal spread of the data points in Figure 2, but also numerically by the $b_{+}$estimate of 0.47 , which is close to the unbiased point of 0.5 .

Having found evidence that people differentially weight various facets of identity leadership when making their evaluations, one important question is how critical this differential weighting is to the success of the explanation provided by the exemplar model. This can be assessed by comparing the results from the model above to a more constrained version of the model that forced equal attention weights across the four dimensions of identity leadership. In this constrained attention implementation of the model, we freely estimated attention weights for authenticity and personal identification dimensions, and constrained the weights for the remaining four social identity dimensions to be equal to one another. This version of the model performed similarly to the flexible attention model in an absolute sense, $R^{2}=0.86$. Enforcing equal attentional weights across the social identity dimensions, best fitting $w_{\mathrm{SI}}=0.22$, also resulted in reduced attention weights for the dimensions of leader authenticity, $w=0.04$, and personal identification, $w=0.07$. The remaining parameter estimates were similar to the original model, $c=9.53, \gamma=2.29$, and $b_{+}=$ 0.47. Using AIC to adjudicate between the constrained and flexible attention models, leads to the flexible attention model being selected (i.e., $\mathrm{AIC}=-418.61$ for the constrained attention 
model vs. -426.40 for the flexible attention model). We conclude that the improvements in fit afforded by unequal attention weights were non-trivial, which reinforces our finding that people differentially weight the various facets of identity leadership when evaluating leader competence.

\section{Analysis of Presidential Effectiveness Data}

In our second analysis, we examine the prototype and exemplar models' abilities to predict presidential effectiveness ratings from category assignments based again on competence ratings. Besides the change in dependent variable, the structure of this analysis is identical to the previous one. We note, however, that analyzing a novel property of a stimulus based on its category membership — or alternatively, estimating membership of one category (that of effective US Presidents) from another category (that of competent leaders) - steers this analysis toward the realm of inductive reasoning (e.g., Osherson, Smith, Wilkie, Lopéz, $\&$ Shafir, 1990). We view our approach as most similar in spirit to the analysis of Heit and Hayes (2011), who developed and explored the relationship between reasoning and familiarity within an exemplar-based computational framework.

We again used the four identity leadership dimensions along with authenticity and identification with an individual as feature dimensions in the prediction of presidential effectiveness ratings. The predictions of the prototype and exemplar models against the data are shown in Figures 8 and 9, respectively. 


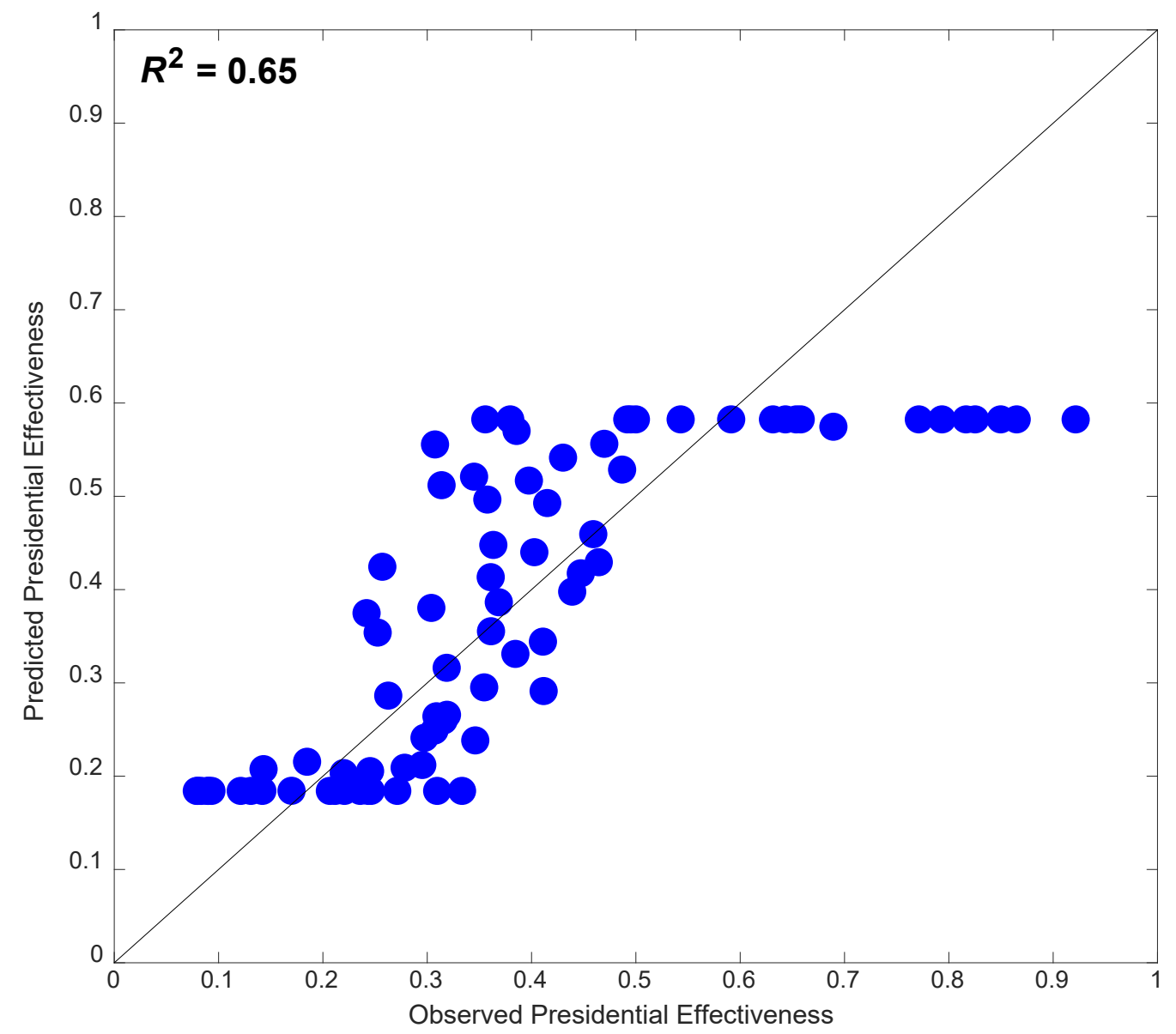

Figure 8. Observed presidential effectiveness ratings plotted against predicted values of the prototype model. Each data point in the plot corresponds to one of the 80 stimuli.

Examining the prototype model predictions, the same issues that arose for the leader competence ratings re-appear for the presidential effectiveness ratings. Specifically, the model qualitatively fails to predict the more extreme-valued ratings (see Figure 8). This is again due to the lack of broad separation of the two prototypes in psychological space, which could not be appropriately offset by increasing the value of the $c$ parameter. Quantitatively, the fit of the prototype model is slightly worse than its fit to the leader competence data. We again conclude that the prototype model fails to provide an adequate account of the data. 


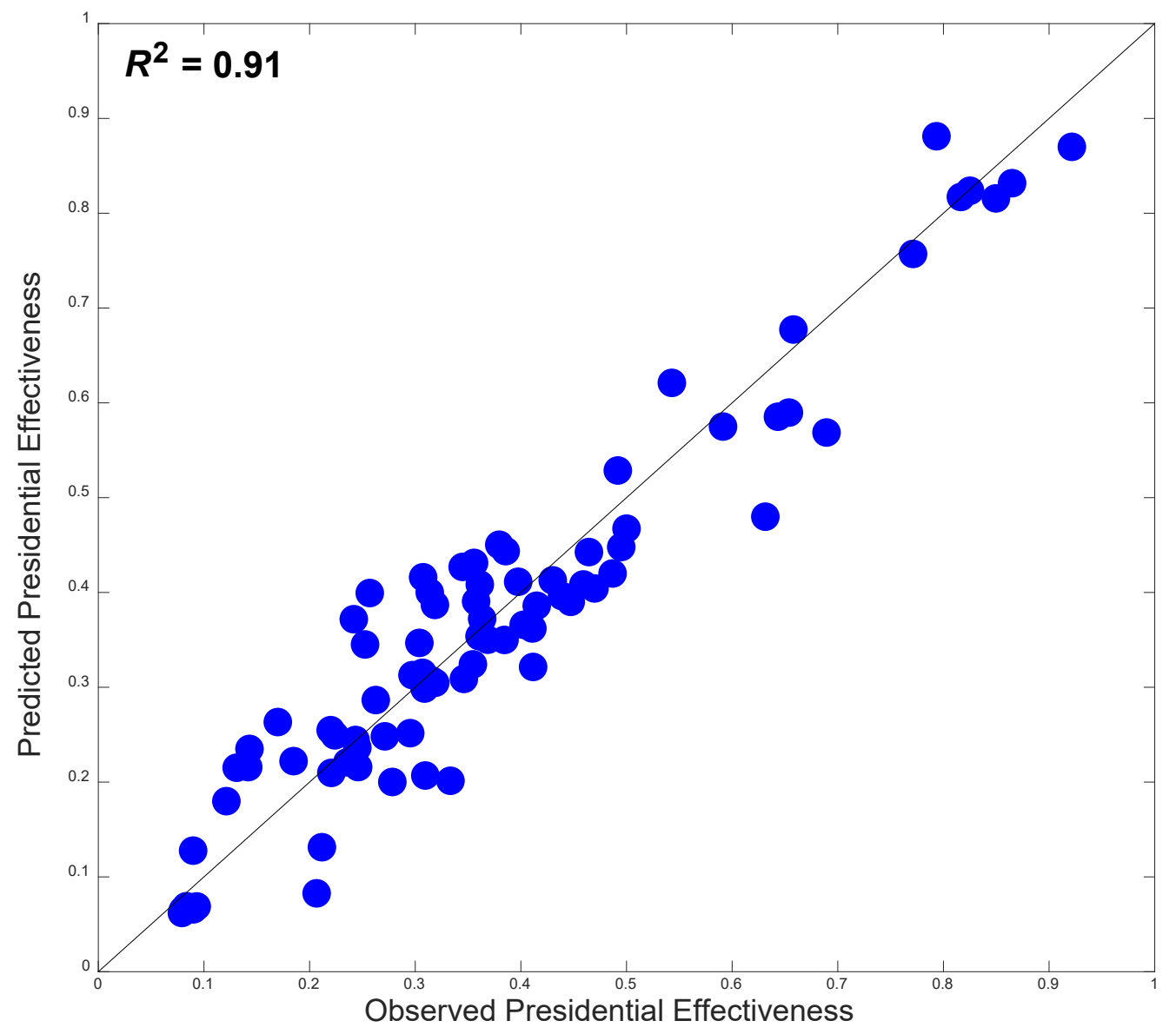

Figure 9. Observed presidential effectiveness ratings plotted against GCM-predicted values. Each data point in the plot corresponds to one of the 80 stimuli.

In contrast to the prototype model, the GCM predictions corresponded very well with the data, $R^{2}=0.91$ (see Figure 9). We think the comparable quality of the overall model fit is especially noteworthy, as the presidential effectiveness data are more heavily concentrated on the lower end of the rating scale compared to leader competence (cf. Figures 3 and 4). We can again use the AIC to formally contrast the fits of the GCM and the prototype model. As 
with the Leader Competence data, the GCM is selected over the prototype model, as it has a lower AIC value (i.e., -431.02 for the GCM vs. -328.34 for the prototype model).

Analysis of the attention weights shown in Table 5 reveals that, once again, only the advancing and embedding dimensions of identity leadership factored into predicting presidential effectiveness, producing respective weights of 0.51 and 0.45 . Across the two ratings, the relative importance of these attributes reversed (i.e., embedding produced a higher attention weight for predicting overall leader competence, but a lower attention weight for presidential effectiveness), suggesting that different attributes may factor more or less heavily depending on the kind of leadership judgment in question (e.g., a specific leadership role, versus leadership ability in general). The only other attribute that contributed to predicting presidential effectiveness was personal identification with the person in question $(w=0.05)$; authenticity no longer conferred any predictive benefit, in contrast to the competence ratings. In terms of response bias, predictions of presidential effectiveness for our stimulus set tended to be pessimistic, with a strong bias away from positive ratings $\left(b_{+}=\right.$ 0.33 , implying a negative response bias; i.e., $\left.b_{-}=0.67\right)$. In practical terms, this response bias implies that on the whole people tended to view the sample of stimuli in this study as rather ineffective US presidents.

We again considered an alternative version of the model that constrained attention weights to be equal across the four dimensions of identity leadership, implemented the same way as for the leader competence data. As with the leader competence data, the constrained model performed similarly to the original model in an absolute sense, $R^{2}=0.88$. Enforcing equal attentional weights across the social identity dimensions, best fitting $w \mathrm{~s}=0.25$, resulting in the remaining attention weights to be estimated to equal 0 . The other parameter 
estimates were similar to the original model, $c=50, \gamma=1.30$, and $b_{+}=0.33$. Using AIC to adjudicate between the constrained and flexible attention models, leads to the flexible attention model being selected (i.e., AIC $=-414.77$ for the constrained attention model vs. 431.02 for the flexible attention model). Again, we conclude that the benefit of allowing attention weights to vary across the dimensions of identity leadership were non-trivial, suggesting that people differentially weight the facets of identity leadership when judging presidential effectiveness.

\section{Comparing Memory Processes Involved in Rating Generic and Specific Leadership}

\section{Roles}

Both the number of dimensions factoring into general versus specific leadership ratings as well as the relative importance of the included dimensions differed. We also note that there appeared to be differences in how information in memory was recruited in making these judgments. Recall that the GCM permits analysis of how broadly exemplar-based information is recruited when making decisions via an analysis of the similarity gradient parameter, $c$. As values for this parameter increase, the model behaves more like a nearestneighbor classifier, insofar that judgments about a target stimulus become dominated by information about the single most-similar exemplar in memory. For example, when $c$ takes on a large value, if a to-be-judged stimulus was most similar to Barack Obama, leadership judgments about this stimulus would be almost exclusively driven by information about Barack Obama. Comparing the values of $c$ for fits to the general leader competence data versus presidential effectiveness data reveals that a far narrower subset of exemplars was relevant for making judgments about presidential effectiveness. That is, the more specific the leadership role — in this case, a potential US president—it appears that effectiveness ratings 
were based almost exclusively on the single most similar exemplar included in our stimulus set. When making judgments about general leader competence, a broader set of exemplars appears to factor into the judgments one makes.

\section{Robustness Analysis of the GCM}

We also considered the robustness of our GCM analysis by conducting two crossvalidation exercises to explore how sensitive the model predictions and parameter estimates were to the composition of the participant data and the number of exemplars used for fitting. These cross-validation exercises are intended to check whether the GCM's fits to the data can be explained in terms of overfitting (e.g., Roberts \& Pashler, 2000; for related discussion see Nosofsky \& Johansen, 2000) and to assess how well the modeling results are likely to generalize to a new dataset. 
Table 6. Model fit statistics and best-fitting parameter estimates for fits of the Generalized Context Model in the two cross-validation exercises. Parameters were estimated by fitting the model to data from odd-numbered raters for each exemplar (Participant Split) or to the complete set of rater data for only odd-numbered exemplars (Exemplar Split). Log likelihood and $R^{2}$ values are based on predictions to the corresponding even-numbered data sets. Parameter estimates for attention weights and response bias are normalized. For the Exemplar Split results, the $n$ used to compute the model likelihood was equal to 40 , as only half the stimuli were used for parameter estimation.

\begin{tabular}{|c|c|c|c|c|c|c|c|c|c|c|c|}
\hline Data Set & $\ln L$ & $R^{2}$ & Embodying & Advancing & Creating & Embedding & Authenticity & Identification & $c$ & $\gamma$ & $b_{+}$ \\
\hline $\begin{array}{l}\text { Participant } \\
\text { Split: Leader } \\
\text { Competence }\end{array}$ & 216.51 & 0.87 & 0.00 & 0.38 & 0.00 & 0.42 & 0.10 & 0.10 & 7.44 & 2.71 & 0.47 \\
\hline $\begin{array}{l}\text { Participant } \\
\text { Split: US } \\
\text { Presidential } \\
\text { Effectiveness }\end{array}$ & 219.827 & 0.90 & 0.00 & 0.38 & 0.00 & 0.48 & 0.00 & 0.14 & 27.65 & 1.47 & 0.33 \\
\hline $\begin{array}{l}\text { Exemplar } \\
\text { Split: Leader } \\
\text { Competence }\end{array}$ & 113.88 & 0.84 & 0.00 & 0.32 & 0.00 & 0.57 & 0.00 & 0.11 & 50.00 & 1.11 & 0.51 \\
\hline $\begin{array}{l}\text { Exemplar } \\
\text { Split: US } \\
\text { Presidential } \\
\text { Effectiveness }\end{array}$ & 115.77 & 0.91 & 0.00 & 0.44 & 0.00 & 0.51 & 0.00 & 0.06 & 50.00 & 1.40 & 0.33 \\
\hline
\end{tabular}

Note. $\operatorname{lnL}=$ Log Likelihood; Identification = Identification with this Individual. 
In the first exercise, we split the participant data into two groups (i.e., even- and oddnumbered participants). Because our participants only rated a small subset of stimuli, the raw rating data were split on a stimulus-by-stimulus basis. For example, there were $N=102$ people who provided ratings for Abraham Lincoln. Data from raters $N=2,4,6$, etc. were assigned to the first group, which the model was fitted to. Data from raters $N=1,3,5$, etc. served as a generalization set. The best-fitting parameter estimates from fitting the model to the first group were fixed, and the resulting predictions for the data from the second group were examined. This method of splitting the data preserves the structure of the original analysis presented above (i.e., the model still has access to the full set of 80 exemplars, and ratings are based on predictions for these 80 stimuli), but effectively reduces the participant sample size by half. By splitting the data in this way, we examine whether the good fit of the GCM was due to overfitting the original sample.

Parameter estimates and fit statistics for the "Participant Split" cross-validation are reported in the top two rows of Table 6 . As can be seen, the fits and parameter estimates are similar to what was found by fitting the model to the full data set. Indeed, the substantive theoretical conclusions we would draw based on this first exercise are virtually identical. The embodying and creating dimensions of social identity theory were not relied upon at all for predicting either leader competence or presidential effectiveness. Ratings appeared to involve some degree of pooling information across multiple exemplars for leader competence ratings, but not for presidential effectiveness, for which ratings appeared to be based on the single most similar exemplar in memory due to the high estimated value for $c$. Overall, the first cross-validation exercise suggests that our original parameter estimates were fairly robust insofar that similar results were achieved by fitting one half of the data, and that the 
predictions remained accurate for (blindly) predicting the second half of the data. Such an outcome is unlikely to occur if the successes of the model were solely due to overfitting and undue flexibility.

In the second exercise, we split the exemplars the model had access to into two groups in an analogous manner. That is, the first group of exemplars were the even-numbered ones such that Adam Sandler, Al Gore, and Barack Obama (and so forth) were exemplars the model had access to for parameter estimation purposes. The second group of exemplars were the odd-numbered ones such that Abraham Lincoln, Albert Einstein, and Arnold Schwarzenegger (and so forth) were the exemplars the model had access to for testing generalization, given the parameter estimates derived from fitting the rating data from the first group of exemplars. This method of splitting the data reduces the number of stimuli by half, but preserves the original number of participants who rated each exemplar. By splitting the data in this way, we can test whether the fits of the exemplar model are reliant on having access to all 80 exemplars in memory, or if a sparser set of leader exemplars can still support accurate model performance. Put another way, withholding access to some exemplars tests whether the good performance of the model is contingent on having access to specific subsets of exemplars. (A further robustness check reported in the Appendix, where we remove stimuli corresponding to fictional and historical figures from the data set yielded largely similar results, and identical conclusions.)

Parameter estimates and fit statistics for the "Exemplar Split" cross-validation are reported in the bottom two rows of Table 6 . Once again, the fits and parameter estimates are similar to what was found by fitting the model to the full data set, with some minor exceptions. The pattern of attention weights is fairly similar, though for leader competence, 
authenticity no longer takes on a non-zero weighting. Regarding the identity leadership dimensions, the embodying and creating dimensions were still assigned weights of zero for predicting leader competence and presidential effectiveness. The only other difference from the original fits concerns the value of $c$ for leader competence. Here, the value is much higher than it was when the model was fit to data with a full complement of 80 exemplars, taking on a similar value to estimates for estimating presidential effectiveness. This is perhaps due to having fewer exemplars in memory. With more exemplars in memory to draw from, pooling information across multiple exemplars likely confers a "smoothing" benefit — akin to averaging - as this information can be pooled across small clusters of similar exemplars. With fewer exemplars in memory, pooling information across a broader range of exemplars may allow for somewhat dissimilar exemplars to disproportionately influence similarity computations. In all other regards, however, the main theoretical conclusions we would draw based on the second cross-validation exercise are the same as the ones based on our primary modeling analysis. We conclude that the second cross-validation analysis shows that the performance of the model is robust against fairly dramatic changes to the exemplars used for parameter estimation purposes, speaking against concerns about overfitting, as the parameter estimates achieved by fitting half of the exemplar data were successful in (blindly) predicting the second half.

\section{Summary of the Modeling Analysis}

The results of our modeling analysis of leadership rating data revealed that some aspects of identity leadership (viz. advancing and embedding a shared sense of 'us') were

highly relevant for predicting leadership judgments in both generic and specific contexts. Notably, the weightings of different dimensions of leadership also differed depending on whether judgments of general leader competence or effectiveness in a specific role were 
being modeled. Overall, we showed that an exemplar-based representation linking the conceptual structure of identity leadership to leadership judgments performed better than a prototype representation based on average feature values. The analysis using the GCM further showed that the breadth of exemplars contributing to these judgments differed quite dramatically, with an extremely narrow subset of exemplars influencing presidential effectiveness ratings, and a broader subset of exemplars influencing judgments of overall leader competence. This last finding shows that contextual factors can impact not just the way features are weighted when making decisions, but also the way information in memory is recruited to support those decisions.

One might be tempted to conclude that the GCM was advantaged in our analysis because it had access to a greater number of representational units (i.e., 79 exemplars vs. two prototypes). We point out that the models are highly similar in terms of the number of free parameters they have access to - a traditional method of assessing model complexity. The exemplar model has only one additional free parameter, which is the response scaling parameter, $\gamma$. We further point out that having access to more representational units is not automatically beneficial. This is because the positioning of exemplars in our study along with the assigned category membership of each exemplar is based on rating data. If category assignment of exemplars were highly irregular, such that exemplars close to one another in psychological space — sharing similar values along attribute dimensions — were not often assigned to the same category, the predictions of the exemplar model would also be irregular, tracking the exemplar-by-exemplar changes in category assignment, leading to category assignment errors. By contrast, prototype representation is less vulnerable to such irregularity, as this "noise" in category assignment of individual exemplars can be averaged out. Overall, we attribute the relatively poor performance of the prototype model to the poor 
correspondence between its summary representations and those used by participants in our study.

\section{Discussion}

In this article, we presented the first analysis of identity leadership that explicitly combines the representational assumptions of psychological models of categorization (i.e., Minda \& Smith, 2011; Nosofsky, 1986; 2011) with the conceptual structure identified using psychometric approaches (i.e., Steffens et al., 2014; van Dick et al., 2018). We showed that an exemplar-based memory representation can provide a highly accurate account of leadership judgments in both the broad sense of overall leader competence as well as in the specific sense of US presidential effectiveness. The success of the exemplar model reinforces some of the core theoretical assumptions of identity leadership. Specifically, the analysis highlighted the importance of the advancing and embedding dimensions (the degree to which a leader is seen to champion shared collective interests and create structures that allow members to live out their shared collective identity). At the same time, the identity leadership dimensions assessing the extent to which a leader is seen as 'one of us' (embodying) and as creating a sense of 'we' and 'us' (creating) did not contribute to judgments of leader competence or US presidential effectiveness. In addition, there were context-dependent (but smaller) contributions relating to leader authenticity and personal identification with a potential leader but these weighted less than the identity leadership dimensions in individuals' overall judgements of leader competence and presidential effectiveness. Methodologically, we have demonstrated that a cognitive modeling approach permits detailed quantitative assessment of a leadership theory (in this case, the social identity theory of 
leadership) that is built upon a psychometric measurement model (i.e., Steffens et al., 2014; van Dick et al., 2018).

This cognitive computational approach to testing theory provides an integrated way of linking conceptual assumptions with psychological processes in a way that has not yet been achieved in the field. Historically, cognitive-oriented theories of leadership (e.g., Epitropaki et al., 2013; Junker \& van Dick, 2014; Lord, Foti, \& De Vader, 1984; Offermann, Kennedy, \& Wirtz, 1994) have been tethered to representational assumptions - typically prototype representations of category knowledge (e.g., Rosch, 1975, 1978; Rosch \& Mervis, 1975)_but had not explicitly tested the adequacy of those assumptions in directly linking theoretical leadership concepts to behavioral data. We hope that the present illustration spurs researchers to test theories of interest in this way. As argued recently by Ballard et al. (in press), quantitative modeling presents a powerful approach to developing and testing theory in organization science that allows for detailed examination of theoretical assumptions (see also Farrell \& Lewandowsky, 2010, 2018; Hintzman, 1991), as well as integration of theory across different levels of analysis (e.g., Sewell \& Smith, 2016). We believe that model-based approaches have much to offer in terms of comparing and further developing existing theories of leadership. Indeed, the approach provides an avenue for integrating different theoretical frameworks by incorporating attribute dimensions derived from different theories (e.g., cognitive-oriented implicit theories of leadership alongside those derived from social identity theory of leadership) to more thoroughly map out the feature space used for representing different categories of leaders. 


\section{Theoretical Implications}

In the present work, we used cognitive modeling to test the psychological assumptions underpinning social identity theorizing of leadership. It was noteworthy that in the present study the extent to which leaders were seen as (a) championing shared national interests (rather than their own interests of those of other outgroups; Haslam \& Platow, 2001; van Knippenberg \& van Knippenberg, 2005) and (b) as capable of embedding group identity in a material reality (Haslam et al., 2011) weighted most strongly in individuals' judgments of leaders' effectiveness. Underscoring the importance of these dimensions in the present study, these two dimensions were found to have a stronger bearing on leadership judgments than alternative predictors as found in leaders' authenticity (Gardner et al., 2011) and individuals' personal identification with the leader (Ashforth et al., 2016). Interestingly, the two identity leadership dimensions that the vast majority of empirical work has focussed on to date (for recent reviews, see Epitropaki et al., 2017; Haslam et al., 2020; van Dick et al., 2018) - relating to the degree to which leaders were seen (a) as being representative of group members and (b) engaging in efforts to craft a sense of us and define what it means to be a member of the group — weighed little in these judgments. These findings provide some evidence of the usefulness of distinguishing between different ways in which leaders can engage with and cultivate shared identity in understanding followers' responses to leaders in particular contexts.

In addition, in light of meta-analytic findings from over a hundred studies (including experimental studies) that reveal that a leader's representativeness of their group — which for simplicity, we referred to as the embodying dimension in the present work-is associated with indicators of leader effectiveness (Barreto \& Hogg, 2017; Steffens et al., 2020), the findings allude to the possibility that the importance of particular dimensions might vary 
across situations and outcomes of interest. We can only speculate why the creating and embodying dimensions of identity leadership (i.e., identity entrepreneurship and group prototypicality) had little impact on judgments of leader competence and Presidential effectiveness in the present study. One potential reason is that, particularly in relation to judgments of Presidential effectiveness, people gravitate more towards leaders' capacity to provide tangible (structural and material) outcomes - as brought about by championing collective interests and initiating concrete actions and policies for the group —in judging candidates as would-be leaders of their nation.

It is also important to note that the present findings do not suggest that there is generally no benefit for leaders to act as identity entrepreneurs (who create an idea of what it means to be a member of the group) or to be seen as in-group prototypes (who embody their group). Indeed, previous research shows that there is not necessarily a straightforward relationship between the factors that influence the effectiveness as a leader and those that influence the tendency to be seen as or to become an effective leader. For example, research has shown that leaders' narcissism and extraversion are factors that influence whether individuals rise to leadership positions but account for much less variance in actual effectiveness once they occupy leadership positions (Grijalva, Harms, Newman, Gaddis, \& Fraley, 2015; Judge, Bono, Ilies, \& Gerhardt, 2002). In a similar vein, it is possible that leaders' identity entrepreneurship and group prototypicality may have little bearing on whether we come to see (or categorize) someone as a leader but nevertheless they may determine the extent to which a person is able to (continue to) influence others (i.e., to perform as a leader). Regardless, the present findings reveal that in explicit judgments of leader effectiveness (i.e., of whether this person is a competent leader in general and would make an effective leader of one's nation), leaders' identity entrepreneurship and 
prototypicality add little above and beyond leaders championing and embedding of shared identity.

By taking theories developed within the factor analytic tradition as providing a dimensional basis for a psychological space for representing different target stimuli, we show that the relative importance of the dimensions that make up the theorized factor structures can be precisely measured across different judgment contexts. We further showed that patterns of rating data were consistent with assumptions of an underlying exemplar-based representation, but inconsistent with a strong form of prototype representation. These findings expand upon discussions of the ways in which we construct categories. In this regard, research within social identity theorizing of leadership has focused on the question of whether social categorization (of individuals in terms of groups) is based on a summary representation encapsulating an average or ideal member (e.g., Halevy, Berson, \& Galinslky, 2001; Steffens, Haslam, Ryan, \& Kessler, 2013; van Knippenberg, 2011) while implicit leadership theory has focussed on the question of whether a leader's overlap with central-tendency versus idealbased categories of leaders in general influence other peoples' reactions to them (Epitropaki et al., 2013; Junker \& van Dick, 2014; van Quaquebeke, Graf, \& Eckloff, 2014). The present work highlights the need to consider not just ideals and central tendencies of abstract categories, but also representations of concrete individuals when theorizing about judgments of leader effectiveness. Indeed, the range of exemplars that factor into judgments may depend on the kind of leader judgment being made. In our data, judgments of a target's US presidential effectiveness were well-described by a model relying on only the most similar exemplars in memory, whereas more general judgments about overall leader effectiveness pooled information from other less similar exemplars. 
Incorporation of exemplar-based representation alongside forms that involve higher degrees of abstraction has also been discussed in the cognitive literature on concepts and categorization (e.g., Allen \& Brooks, 1991; Erickson \& Kruschke, 1998) with exemplars providing a context-dependent means of coordinating and gating access to more abstract summary information about categories (Sewell \& Lewandowsky, 2011, 2012). We hasten to point out that the success of the exemplar model in our study does not invalidate or speak against the need to incorporate ideals and summary representations in leadership theorizing. Rather, we note that our results indicate the utility of incorporating exemplar-based information into leadership theories, especially with regards to highly specific leadership roles, where judgments appear to be strongly driven by a small number of exemplars in memory. Indeed, while there are different ways of conceptualizing ideal category members, previous analyses do not specify what it is that represents ideal leaders or group members (what makes an ideal leader or group member?) and, in this regard, it seems that exemplarbased representations may help shed light on this question (see Voorspoels et al., 2011, for theoretical discussion). For example, judgments about ideal category members may in part derive from resemblance to highly salient exemplars. Consistent with our findings revealing superior fit of an exemplar-based representation over a prototype representation, this may also explain in part previous findings that ideal group members (and ideal leaders) tend to evoke more support than average or typical group members (and average or typical leaders) (e.g., Halevy et al., 2011; van Quaquebeke et al., 2014; Steffens et al., 2020). Having said this, there are other ways of construing prototype representation that have been advocated for in regards to leadership theories and the more general literature on concept and category representation that may prove more successful than the "feature average" implementation we pursued in this article. We discuss some of these other formulations, including potential challenges and opportunities for future work, below. 


\section{Limitations and Future Directions}

We now discuss potential limitations of the current study that should be addressed in future research. First, we note that our theoretical inferences are all based on interpreting fits of cognitive models to data. As these models have been developed to provide accounts of the underlying cognitive processes that support categorization, they are causal in nature.

However, because our use of the models in this instance is with rating scale data rather than experimental data, there are sensible concerns about endogeneity (e.g., Antonakis, 2017; Antonakis, Bendahan, Jacquart, \& Lalive, 2014). In particular, it is prudent to keep in mind the limitations of what is revealed by a good fit to data. While we are inclined to accept the causal account of the data provided by the GCM — that is, that leadership judgments are based on the similarity between a candidate leader and competent (and incompetent) leader exemplars in memory - there is an important caveat. As with any cognitive modeling analysis, a good fit to data alone cannot be viewed as validation of the underlying causal assumptions that are built into a model, nor can it be viewed as proof that the model is "correct" in the sense that it reflects the true underlying data-generating process. Rather, a good fit to data only shows that the causal assumptions outlined by the model provide a plausible account of the current data set. It is possible, perhaps even probable, that there are alternative models — engendering other potentially conflicting causal assumptions - that might provide equally compelling (or better) accounts of the data. However, it behoves researchers to develop and test these alternative theoretical models in order to explicitly demonstrate their viability. To the extent that one model is able to outperform existing competitors and fit a wide range of data, one can be confident that the causal account provided by that model is acceptable for understanding and interpreting data. On this point, we emphasize the utility of comparing different theoretical frameworks for leadership across 
contexts through the lens of cognitive models such as the GCM, as the adequacy of different dimensional and processing assumptions can be contrasted via competitive fits to data. We believe our study is a good example of this utility, as we (1) contrasted two alternative models of category representation against one another, (2) incorporated alternative predictors to those specified by social identity theory (viz. authenticity and personal identification with the leader), and (3) assessed the adequacy of the representational assumptions across two different leadership contexts.

One issue to consider is whether cognitive processes and/or representations that are engaged vary across a range of more or less consequential decisions and behaviors (e.g., Hertwig \& Orttman, 2001; Podsakoff \& Organ, 1986). Our study involved seemingly nonconsequential decisions in that people were rating the presidential effectiveness of (say) Bill Gates in a hypothetical fashion with no real-world ramifications. It remains to be seen to what extent other consequential decisions — such as casting a vote — might engage a similar mode of processing, weighting of information, or level of abstraction used to construe category information. Introducing a way of having people's decisions/ratings produce personal consequences (e.g., via different reward incentives associated with different leaders) might help shed light on how category information is used (if at all) when people's decisions have clear and direct personal ramifications. Such incentives have been shown to alter fundamental aspects of simple decision-making processes (e.g., Ballard, Sewell, Cosgrove, \& Neal, 2019). Extending this idea further, it is possible to use the models developed here to predict historical (and future) election results, including situations involving novel candidates. Doing so would require collection of relevant attribute data for the candidates in question, as these form the basis of the similarity comparisons that drive responding (cf. Antonakis \& Dalgas, 
2009). We leave more detailed development of this idea to future research, as it requires careful consideration of methodological issues beyond the scope of the current work.

A general theoretical question raised by our work is whether our results constitute evidence against prototype representation. We wish to be clear in stating that our results provide support for exemplar-based representation as an alternative to the strong form of prototype representation - typical of the categorization literature - that we implemented in our modeling. This does not, however, imply that some form of prototype (or prototype-like) representation is incorrect. As mentioned earlier, there are many other theoretically viable ways of developing the prototype model that we did not consider here, some of which may fare better than a pure exemplar-based representation. The most common computational interpretation of a prototype is that of an average across all exemplars within a category (e.g., Nosofsky, 1992a; Minda \& Smith, 2011; Reed, 1972), and so we viewed this as a suitable starting point for comparing the two classes of representations in a rigorous way. However, prototypes can also be conceptualized in terms of a modal set of features (rather than an average), or even an idealized set of features that need not correspond to any central tendency summary of exemplars (see Voorspoels et al., 2011, for theoretical discussion along these lines).

Indeed, there is a continuum of abstraction that relates prototype- and exemplar-based representation, and some theories characterize prototypes in this way, (e.g., as a collection of multiple prototypes that abstract across small subsets of locally similar exemplars, as in the SUSTAIN model of Love et al., 2004). This perspective is more strongly aligned with the notion of hierarchical organization of concepts, as articulated by Rosch and colleagues (e.g., Rosch, 1975, 1978; Rosch \& Mervis, 1975). As an example, one can imagine a singular 
summary representation of the category of FURNITURE, but one can also consider more fine-grained summary representations or a subset of exemplars that are nested within this higher-order category (e.g., TABLES). Indeed, finer-grained levels of organization remain possible (e.g., KITCHEN TABLES vs. OFFICE TABLES). With respect to leadership, prototypes are often considered at some intermediate level of abstraction (e.g., BUSINESS LEADERS vs. POLITICAL LEADERS; Lord et al., 1984), though higher levels of abstraction are also commonly considered (e.g., Epitropaki et al., 2013; Junker \& Van Dick, 2014).

A target for future research is to develop and test more refined versions of prototype representation that explicitly model category substructure. However, we believe that doing so requires overcoming some theoretical and computational challenges. Computationally, it is challenging to choose an appropriate number of intermediate-level prototypes, as this requires determining how exemplars are grouped into sub-categories in a theoretically principled way. The variable abstraction model of Vanpaemel and Storms (2008) is one attempt to achieve this, but is computationally expensive, as it relies on model selection to test all possible ways of partitioning a set of $N$ stimuli (or individuals, in the leadership context) into sub-groups. The number of potential sub-groupings to consider quickly becomes prohibitive, as the number of stimuli increases, and is given by the $N$ th Bell number. For example, there are 21,147 ways to partition a set of 10 stimuli into a variable number of groups, and $190,899,322$ ways to partition a set of 15 stimuli. It would be impossible to apply such a model to the 80 -stimulus set we used here. A theoretical challenge lies in motivating the assignment of exemplar subsets into sub-categories, as this requires making additional assumptions about sub-group membership. With the real-world target stimuli we considered here, such assignments are not necessarily clear-cut (e.g., Arnold Schwarzenegger and 
Ronald Reagan could be regarded as both actors and politicians; Bill Gates could be regarded as a business person and philanthropist; Donald Trump could be regarded as a business person, politician, and reality television personality, among other things). For dealing with group-level data, assignment of exemplars to sub-categories could be empirically justified (e.g., based on the rate at which an individual is assigned to a particular sub-category). For dealing with individual-level data, assignments could be specific to each individual. In either case, the rationale for grouping stimuli in a way that supports intermediate-level prototype representation needs to be carefully developed. One possible way of addressing these concerns in a principled and empirically-motivated fashion would be to use a category construction task to identify subsets of exemplars that cohere with one another, and are viewed as distinct from other subsets of exemplars by participants. Such an approach might help clarify the kinds of sub-categories people rely on when making leader decisions, and could be used to determine the number and relative positioning of different prototypes people use to represent a range of leader sub-categories. This kind of representation could be incorporated into a GCM-like modeling framework, where leader judgments are driven by relative similarity to multiple prototypes rather than exemplars. Evaluating such a model against the exemplar-based model is an exciting theoretical prospect for future research.

Following on from the above, there is the possibility that some judgments might be more amenable to exemplar (or prototype) representation than others. For example, people might rely more heavily on exemplar-based representations when considering more specific leadership roles, and on more abstract prototype-like representations when considering less well-defined roles (or when a reference category is specified at a higher or lower level of abstraction). While our results suggest that an exemplar-based representation adequately accounts for assessments of both general and specific roles, it is important to keep in mind 
that we collected ratings about both roles from the same individuals. Gathering ratings about either a specific or general role might lead people to engage in different modes of processing that entail accessing representations at different levels of abstraction, though this did not appear to be the case in our data set.

Another factor, that applies equally to exemplar and prototype models, concerns the number of contrast categories (or sub-categories) that are considered when evaluating a particular individual. It is possible — perhaps even likely_that some individuals will be evaluated against different subsets of exemplars (or prototypes), or that people's judgments may be informed only by information about a single category (e.g., Murphy \& Ross, 1994). The models we have presented here could be developed in ways that allow for judgments to be made in this way. For example, a thresholded single-category similarity rule could be used for decision-making. Alternatively, one could assume that control of the decision process is based on a winner-take-all competition among representational units, as in the SUSTAIN model of Love et al. (2004). In any case, the justification for such a decision rule must be appropriately developed. For example, there must be compelling evidence for why a particular individual is evaluated against exemplars $\mathrm{A}, \mathrm{B}$, and $\mathrm{C}$, but not $\mathrm{D}, \mathrm{E}$, and $\mathrm{F}$, and why another individual might be evaluated against a different subset of exemplars. The necessity of such assumptions must be considered against the success of the more parsimonious assumption that we have instantiated in our modeling: that each individual is evaluated against the same set of exemplars (or prototypes).

Furthermore, we note that the rating data that we collected-which determined the positioning of exemplars and prototypes in psychological space-were averaged across a number of different individuals. That is, different individuals contributed data about different 
stimuli. Consequently, we can only interpret the positioning of exemplars and prototypes in terms of what might characterize a "typical" individual. To get a clearer sense of the relative adequacy of prototype and exemplar-based representations, it would be ideal - though perhaps practically challenging - to collect more comprehensive rating data from participants. If a participant were to provide rating data for all stimuli of a given set, we could use this information to position exemplars and category prototypes such that they accurately capture the attribute ratings that are unique to that participant. In the domain of perceptual categorization, such calibration is often achieved using multi-dimensional scaling, which relies on collecting a large quantity of pairwise similarity ratings (e.g., Nosofsky, 1992b; Nosofsky et al., 2018). With large stimulus sets, such as the one we used here, this approach is somewhat impractical. However, having validated the underlying dimensional structure using the factor analytic approach circumvents this challenge, making model comparison at the level of individual participants viable. An upshot of this approach is that it permits detailed analysis of individual differences, both in terms of how people represent leader exemplars (or prototypes) but also in terms of how different people might weight the different leadership attributes when making judgments in different contexts. Mapping out potential patterns of such variation is a clear target for future research.

Another issue with the rating data mentioned earlier in the article concerns commonsource bias (e.g., Avolio et al., 1991; Dionne et al., 2002; Podsakoff, MacKenzie, Lee, \& Podsakoff, 2003). We discussed in the Method section how application of the GCM and prototype models and their interpretation as psychological theories of leader categorization is reliant on having the same individuals rate both feature and outcome attributes. We now discuss several other reasons why common-source bias might not be especially problematic for our study. First, common-source bias implies that there might be dependencies in the way 
stimulus attributes are coded. Computationally, this implies that assuming psychologically separable dimensions would be inappropriate, and that similarity would instead have to be computed using a Euclidean distance metric. We examined this issue by comparing the fits of the models (and theoretical conclusions) using both city-block and Euclidean distance metrics, which respectively map onto assumptions about psychological separability and integrality (Garner, 1974; Nosofsky, 1986). We found that both distance metrics produced similar fits, parameter estimates, and resulted in the same theoretical conclusions, suggesting some degree of robustness against the common-source bias (see Appendix). Moreover, the best-fitting set of attention weights for both leader competence and presidential effectiveness was highly uneven across dimensions (see Table 5), suggesting that some attribute dimensions were effectively irrelevant for making these judgments, speaking against the idea of mutual influence.

Finally, a surprising finding of the current study was that the embodying and creating dimensions of identity leadership appeared unrelated to formulating judgments of leader competence as well as US Presidential effectiveness. An open question is whether this reflects something general about the utility of these dimensions for these particular judgments, or if this reflects peculiarities about our specific set of stimuli or the specific leadership roles considered in our study. Applying a model-based analysis to a wider range of potential leadership roles, especially a broader range of specific roles (e.g., the attributes that are important for predicting presidential effectiveness may differ dramatically from the attributes that are important for predicting effectiveness as a coach for a children's soccer team) will help clarify when different identity leadership attributes factor into people's judgments. Similarly, references to 'competent', 'effective', or 'good' leaders may seem synonymous but it is possible that there may be subtle variation in people's responses as a 
function of different meanings we associate with different reference categories. Finally, developing different stimulus sets comprising different individual identities (including less US-centric sets of individuals) will help clarify how sensitive patterns of attention weights are to the composition of the stimulus set.

An outstanding question not addressed by our modeling concerns the time course of leader categorization. The model-based analysis we presented only addresses choice behavior, neglecting both encoding and response dynamics, and addressing these factors is an ambitious target for future research. Our study was not designed to explore these dynamics, as we allowed participants an unlimited amount of time to rate each of the stimulus identities. It is therefore prudent to assume that our participants had access to information along each attribute dimension when rating leader competence and presidential effectiveness. However, the models we have investigated here are amenable to being extended in ways that permit analysis of these dynamics. For example, on the encoding side, the extended GCM of Lamberts (1995, 1998; see also Cohen \& Nosofsky, 2003) addresses encoding dynamics by incorporating a time-dependent similarity function. This accounts for people having access to some attributes sooner than they have access to others. On the decision-making side, the exemplar-based random walk model of Nosofsky and Palmeri (1997) explains categorization response times by modeling decision-making as an evidence accumulation process driven by retrieval of exemplars from memory. Work by Sewell and colleagues has examined how exemplar-based associative information in memory can be used to model decision dynamics over the course of learning (e.g., Sewell, Jach, Boag, \& Van Heer, 2018; Sewell \& Stallman, 2020). Additional work by Lamberts (2000) has shown how both encoding and decision dynamics can be incorporated into a single model to explain how decision dynamics continuously change as additional stimulus information becomes available. We believe a 
complete understanding of how time-based factors affect leader categorization can be achieved by a combination of careful experimentation (e.g., incorporating manipulations of time pressure and time-limited encoding) and cognitive modeling, supplemented with analysis of people's subjective reports of their decision strategies and attribute weightings.

\section{Conclusions}

We presented a cognitive modeling approach to testing the assumptions of the social identity theory of leadership. By combining the dimensional structure outlined by social identity theory of leadership with cognitive models of categorization, we were able to contrast the descriptive adequacy of different theoretical assumptions. Using rating data for both general and specific leadership roles, we were able to show that an exemplar-based representation (i.e., where decisions are based on similarity to highly salient exemplary leaders) provided a better account of both sets of data than one form of traditional prototypebased representation (i.e., based on similarity to the average across the entire set of all leaders). We further showed that judgments about effective leaders were primarily driven by advancing and embedding dimensions of identity leadership (i.e., would-be leaders' championing 'our' collective interests and embedding structures to turn the idea of 'us' into a material reality), while embodying and creating dimensions (as well as leader authenticity and respondents' personal identification with the leader) were largely irrelevant to judgments of leader effectiveness. These patterns were largely consistent across judgments of leadership in both general (i.e., whether a person makes a competent leader) and in a specific role (i.e., that of the US president). The cognitive modeling approach illustrated here provides a novel way of extending social identity theorizing of leadership that enables rigorous testing of the underlying processes. Our results suggest that the cognitive basis for seeing individuals as 
effective leaders resides not so much in their similarity to a collective embodiment of category members as it does in their capacity to live up to the best exemplars of 'us'. 


\section{References}

Akaike, H. (1974). A new look at the statistical model identification. IEEE Transactions on Automatic Control, 19, 716-723.

Allen, S. W. \& Brooks, L. R. (1991). Specializing the operation of an explicit rule. Journal of Experimental Psychology: General, 120, 3-19.

Alvesson, M. \& Einola, K. (2019). Warning for excessive positivity: Authentic leadership and other traps in leadership studies. The Leadership Quarterly, 30, 383-395.

Anderson, J. R. (1991). The adaptive nature of human categorization. Psychological Review, 98, 409-429.

Antonakis, J. (2017). On doing better science: From thrill of discovery to policy implications. The Leadership Quarterly, 28, 5-21.

Antonakis, J., Bendahan, S., Jacquart, P., \& Lalive, R. (2014). Causality and endogeneity: Problems and solutions. In D. V. Day (Ed.), Oxford library of psychology. The Oxford handbook of leadership and organizations (pp. 93-117). Oxford University Press.

Antonakis, J., \& Dalgas, O. (2009). Predicting elections: Child's play! Science, 323, $1183-$ 1183.

Ashforth, B. E., Schinoff, B. S., \& Rogers, K. M. (2016). "I identify with her," "I identify with him": Unpacking the dynamics of personal identification in organizations. The Academy of Management Review, 41, 28-60.

Avoilio, B. J., Yammarino, F. J., \& Bass, B. M. (1991). Identifying common methods variance with data collected from a single source: An unresolved sticky issue. Journal of Management, 17, 571-587.

Ballard, T., Palada, H., Griffin, M., \& Neal, A. (in press). An Integrated Approach to Testing Dynamic, Multilevel Theory: Using Computational Models to Connect Theory, Model, and Data. Organizational Research Methods.

Ballard, T., Sewell, D. K., Cosgrove, D., \& Neal, A. (2019). Information processing under reward versus under punishment. Psychological Science, 30, 757-764.

Barreto, N. B. \& Hogg, M. A. (2017). Evaluation of and support for group prototypical leaders: a meta-analysis of twenty years of empirical research. Social Influence, 12, 41-55.

Bass, B. M., \& Riggio, R. E. (2006). Transformational leadership (2nd ed.). Erlbaum.

Blader, S. L., \& Tyler, T. R. (2009). Testing and extending the group engagement model: Linkages between social identity, procedural justice, economic outcomes, and extrarole behavior. Journal of Applied Psychology, 94, 445.

Chrobot-Mason, D., Gerbasi, A., \& Cullen-Lester, K. L. (2016). Predicting leadership relationships: The importance of collective identity. The Leadership Quarterly, 27, 298-311. 
Cohen, A. L. \& Nosofsky, R. M. (2003). An extension of the exemplar-based random-walk model to separable-dimension stimuli. Journal of Mathematical Psychology, 47, 150165.

Dinh, J. E., Lord, R. G., Gardner, W. L., Meuser, J. D., Liden, R. C., \& Hu, J. (2014). Leadership theory and research in the new millennium: Current theoretical trends and changing perspectives. The Leadership Quarterly, 25, 36-62.

Dionne, S.D., Yammarino, F.J., Atwater, L.E., \& James, L.R. (2002). Neutralizing substitutes for leadership theory: Leadership effects and common-source bias. Journal of Applied Psychology, 87, 454-464.

Ellemers, N., de Gilder, D., \& Haslam, S. A. (2004). Motivating individuals and groups at work: A social identity perspective on leadership and group performance. Academy of Management Review 29, 459-478.

Epitropaki, O., Kark, R., Mainemelis, C., \& Lord, R. G. (2017). Leadership and followership identity processes: A multilevel review. The Leadership Quarterly, 28, 104-129.

Epitropaki, O., Sy, T., Martin, R., Tram-Quon, S., \& Topakas, A. (2013). Implicit leadership and followership theories "in the wild": Taking stock of information-processing approaches to leadership and followership in organizational settings. The Leadership Quarterly, 24, 858-881.

Erickson, M. A. \& Kruschke, J. K. (1998). Rules and exemplars in category learning. Journal of Experimental Psychology: General, 127, 107-140.

Farrell, S. \& Lewandowsky, S. (2010). Computational models as aids to better reasoning in psychology. Current Directions in Psychological Science, 19, 329-335.

Farrell, S., \& Lewandowsky, S. (2018). Computational modeling of cognition and behavior. Cambridge University Press.

Gardner, W. L., Cogliser, C. C., Davis, K. M., \& Dickens, M. P. (2011). Authentic leadership: A review of the literature and research agenda. The Leadership Quarterly, $22,1120-1145$.

Garner, W. R. (1974). The processing of information and structure. Wiley.

Graen, G. B., \& Uhl-Bien, M. (1995). Relationship-based approach to leadership: Development of leader-member exchange (LMX) theory of leadership over 25 years: Applying a multi-level multi-domain perspective. The Leadership Quarterly, 6, 219247.

Grijalva, E., Harms, P. D., Newman, D. A., Gaddis, B. H., \& Fraley, R. C. (2013). Narcissism and leadership: A meta-analytic review of linear and nonlinear relationships. Personnel Psychology, 68, 1-47.

Hais, S. C., Hogg, M. A., \& Duck, J. M. (1997). Self-categorization and leadership: Effects of group prototypicality and leader stereotypicality. Personality \& Social Psychology Bulletin, 23, 1087-1099. 
Haslam, S. A., \& Platow, M. J. (2001). The link between leadership and followership: How affirming social identity translates vision into action. Personality \& Social Psychology Bulletin, 27, 1469-1479.

Haslam, S. A., Powell, C., \& Turner, J. (2000). Social identity, self-categorization, and work motivation: rethinking the contribution of the group to positive and sustainable organisational outcomes. Applied Psychology, 49, 319-339.

Haslam, S. A., Reicher, S. D., \& Platow, M. J. (2011). The new psychology of leadership: Identity, influence and power. Psychology Press.

Haslam, S. A., Reicher, S. D., \& Platow, M. J. (2020). The new psychology of leadership: Identity, influence and power ( $2^{\text {nd }} \mathrm{Ed}$.). Routledge.

Halevy, N., Berson, Y., \& Galinsky, A. D. (2011). The mainstream is not electable: When vision triumphs over representativeness in leader emergence and effectiveness. Personality \& Social Psychology Bulletin, 37, 893-904.

Heit, E. \& Hayes, B. K. (2011). Predicting reasoning from memory. Journal of Experimental Psychology: General, 140, 76-101.

Hertwig, R \& Ortmann, A. (2001). Experimental practices in economics: A methodological challenge for psychologists? Behavioral \& Brain Sciences, 24, 383-451.

Hintzman, D. L. (1991). Why are formal models useful in psychology? In W. H. Hockley \& S. Lewandowsky (Eds.) Relating theory and data: Essays in human memory in honor of Bennet B. Murdock (pp. 39-56). Erlbaum.

Hogg, M. A. (2001). A social identity theory of leadership. Personality \& Social Psychology Review, 5, 184-200.

Hogg, M. A., van Knippenberg, D., \& Rast, D. E. III. (2012). The social identity theory of leadership: Theoretical origins, research findings, and conceptual developments. European Review of Social Psychology, 23, 258-304.

Hopkins, N., \& Reicher, S. (1996). The construction of social categories and processes of social change: Arguing about national identities. In G. M. Breakwell \& E. Lyons (Eds.), International series in social psychology. Changing European identities: Social psychological analyses of social change, (pp. 69-93). Butterworth-Heinemann.

Judge, T. A., Bono, J. E., Ilies, R., \& Gerhardt, M. W. (2002). Personality and leaderhip: A qualitative and quantitative review. Journal of Applied Psychology, 87, 765-780.

Junker, N. M., \& van Dick, R. (2014). Implicit theories in organizational settings: A systematic review and research agenda of implicit leadership and followership theories. The Leadership Quarterly, 25, 1154-1173.

Kaufman, S. B. (2019, June 15). Authenticity under fire. Scientific American. Retrieved from https://blogs.scientificamerican.com/beautiful-minds/authenticity-under-fire/

Lamberts, K. (1995). Categorization under time pressure. Journal of Experimental Psychology: General, 124, 161-180. 
Lamberts, K. (1998). The time course of categorization. Journal of Experimental Psychology: Learning, Memory, \& Cognition, 24, 695-711.

Lamberts, K. (2000). Information-accumulation theory of speeded categorization. Psychological Review, 107, 227-260.

Lee, E.-S., Park, T.-Y., \& Koo, B. (2015). Identifying organizational identification as a basis for attitudes and behaviors: A meta-analytic review. Psychological Bulletin, 141, 1049-1080.

Lee, M. D. \& Navarro, D. J. (2002). Extending the ALCOVE model of category learning to featural stimulus domains. Psychonomic Bulletin \& Review, 9, 43-58.

Lord, R. G., Foti, R. J., \& De Vader, C. L. (1984). A test of leadership categorization theory: Internal structure, information processing, and leadership perceptions. Organizational Behavior \& Human Performance, 34, 343-378.

Lord, R. G., Foti, R. J., \& Phillips, J. S. (1982). A theory of leadership categorization. In J. G. Hunt, U. Sekaran, \& C. Schriesheim (Eds.). Leadership: Beyond establishment views, pp. 104-121. Southern Illinois University Press.

Love, B. C., Medin, D. L., \& Gureckis, T. M. (2004). SUSTAIN: A network model of category learning. Psychological Review, 111, 309-332.

Luce, R. D. (1959). Individual choice behavior. Wiley.

McGarty, C. (1999). Categorization in social psychology. London: Sage.

Medin, D. L. \& Schaffer, M. M. (1978). Context theory of classification learning. Psychological Review, 85, 207-238.

Minda, J. P. \& Smith, J. D. (2011). Prototype models of categorization: basic formulation, predictions, and limitations. In E. M. Pothos \& A. J. Wills (Eds.), Formal approaches in categorization (pp. 40-64). Cambridge: Cambridge University Press.

Murphy, G. L. (2002). The big book of concepts. MIT Press.

Murphy, G. L. \& Ross, B. H. (1994). Predictions from uncertain categorizations. Cognitive Psychology, 27, 148-193.

Navarro, D. J. \& Lee, M. D. (2003). Combining dimensions and features in similarity-based representations. In S. Becker, S. Thrun, \& K. Obermayer (Eds.), Advances in neural information processing systems (Vol. 15, pp. 67-74). MIT Press.

Neider, L. L., \& Schriesheim, C. A. (2011). The authentic leadership inventory (ALI): Development and empirical tests. The Leadership Quarterly, 22, 1146-1164.

Nelder, J. A. \& Mead, R. (1965). A simplex method for function minimization. Computer Journal, 7, 308-313.

Nosofsky, R. M. (1986). Attention, similarity, and the identification-categorization relationship. Journal of Experimental Psychology: General, 115, 39-57. 
Nosofsky, R. M. (1992a). Exemplars, prototypes, and similarity rules. In A. F. Healy, S. M. Kosslyn, \& R. M. Shiffrin (Eds.), From learning theory to connectionist theory: Essays in honor of William K. Estes (Vol. 1, pp. 149-167). Erlbaum.

Nosofsky, R. M. (1992b). Similarity scaling and cognitive process models. Annual Review of Psychology, 43, 25-53.

Nosofsky, R. M. (2000). Exemplar representation without generalization? Comment on Smith and Minda's (2000) "Thirty categorization results in search of a model." Journal of Experimental Psychology: Learning, Memory \& Cognition, 26, 1735-1743.

Nosofsky, R. M. (2011). The generalized context model: an exemplar model of classification. In E. M. Pothos \& A. J. Wills (Eds.), Formal approaches in categorization (pp.1839). Cambridge University Press.

Nosofsky, R. M. \& Johansen, M. K. (2000). Exemplar-based accounts of "multiple-system" phenomena in perceptual categorization. Psychonomic Bulletin \& Review, 7, 375-402.

Nosofsky, R. M., Little, D. R., \& James, T. W. (2012). Activation in the neural network responsible for categorization and recognition reflects parameter changes. Proceedings of the National Academy of Sciences, 109, 333-338.

Nosofsky, R. M. \& Palmeri, T. J. (1997). An exemplar-based random walk model of speeded classification. Psychological Review, 104, 266-300.

Nosofsky, R. M., Sanders, C., Meagher, B. J., \& Douglas, B. J. (2018). Toward the development of a feature-space representation for a complex natural category domain. Behavior Research Methods, 50, 530-556.

Nosofsky, R. M. \& Zaki, S. R. (1998). Dissociations between categorization and recognition in amnesic and normal individuals: An exemplar-based interpretation. Psychological Science, 9, 247-255.

Nosofsky, R. M. \& Zaki, S. R. (2002). Exemplar and prototype models revisited: Response strategies, selective attention, and stimulus generalization. Journal of Experimental Psychology: Learning, Memory, \& Cognition, 28, 924-940.

Offermann, L. R., Kennedy, J. K., \& Wirtz, P. W. (1994). Implicit leadership theories: Content, structure, and generalizability. The Leadership Quarterly, 5, 43-58.

Osherson, D. N., Smith, E. E., Wilkie, O., López, A., \& Shafir, E. (1990). Category-based induction. Psychological Review, 97, 185-200.

Phillips, J. S. (1984). The accuracy of leadership ratings: A cognitive categorization perspective. Organizational Behavior \& Human Performance, 33, 125-138.

Pillai, R., Williams, E. A., Lowe, K. B., \& Jung, D. I. (2003). Personality, transformational leadership, trust, and the 2000 US presidential vote. The Leadership Quarterly, 14, 161-192. 
Platow, M. J., Haslam, S. A., Reicher, S. D., \& Steffens, N. K. (2015). There is no leadership if no-one follows: Why leadership is necessarily a group process. International Coaching Psychology Review, 10, 20-37.

Platow, M. J. \& van Knippenberg, D. (2001). A social identity analysis of leadership endorsement: The effects of leader ingroup prototypicality and distributive intergroup fairness. Personality \& Social Psychology Bulletin, 27, 1508-1519.

Podsakoff, P. M., MacKenzie, S. B., Lee, J.-Y., \& Podsakoff, N. P. (2003). Common method biases in behavioral research: A critical review of the literature and recommended remedies, Journal of Applied Psychology, 88, 879-903.

Podsakoff, P. M., \& Organ, D. W. (1986). Self-reports in organizational research: Problems and prospects. Journal of Management, 12(4), 531-544.

Posner, M. I. \& Keele, S. W. (1968). On the genesis of abstract ideas. Journal of Experimental Psychology, 77, 353-363.

Postmes, T., Haslam, S. A., \& Jans, L. (2013). A single-item measure of social identification: reliability, validity, and utility. British Journal of Social Psychology, 52, 597-617.

Pothos, E. M. \& Wills, A. J. (2011). Formal approaches in categorization. Cambridge.

Reed, S. K. (1972). Pattern recognition and categorization. Cognitive Psychology, 3, 382-407.

Rehder, B. \& Hoffman, A. B. (2005). Thirty-something categorization results explained: Selective attention, eyetracking, and models of category learning. Journal of Experimental Psychology: Learning, Memory, \& Cognition, 31, 811-829.

Reicher, S. D., Haslam, S. A., \& Hopkins, N. (2005). Social identity and the dynamics of leadership: Leaders and followers as collaborative agents in the transformation of social reality. The Leadership Quarterly, 16, 547-568.

Reicher, S. D., \& Hopkins, N. (2001). Self and nation: Categorization, contestation, and mobilisation. Sage.

Roberts, S. \& Pashler, H. (2000). How persuasive is a good fit? A comment on theory testing. Psychological Review, 107, 358-367.

Rosch, E. (1975). Cognitive representations of semantic categories. Journal of Experimental Psychology: General, 104, 192-233.

Rosch, E. (1978). Principles of categorization. In E. Rosch \& B. B. Lloyd (Eds.) Cognition and categorization, (pp. 27-48). Erlbaum.

Rosch, E., \& Mervis, C. B. (1975). Family resemblances: Studies in the internal structure of categories. Cognitive Psychology, 7, 573-605.

Rosch, E., Mervis, C. B., Gray, W. D., Johnson, D. M., \& Boyes-Braem, P. (1976). Basic objects in natural categories. Cognitive Psychology, 8, 382-439. 
Sewell, D. K., Jach, H. J., Boag, R. J., \& Van Heer, C. A. (2019). Combining error-driven models of associative learning with evidence accumulation models of decisionmaking. Psychonomic Bulletin \& Review, 26, 868-893.

Sewell, D. K. \& Lewandowsky, S. (2011). Restructuring partitioned knowledge: The role of recoordination in category learning. Cognitive Psychology, 62, 81-122.

Sewell, D. K. \& Lewandowsky, S. (2012). Attention and working memory capacity: Insights from blocking, highlighting, and knowledge restructuring. Journal of Experimental Psychology: General, 141, 444-469.

Sewell, D. K., \& Smith, P. L. (2016). The psychology and psychobiology of simple decisions: Speeded choice and its neural correlates. In C. Montag and M. Reuter (Eds.) Neuroeconomics, (pp. 253-292). Springer.

Sewell, D. K. \& Stallman, A. (2020). Modeling the effect of speed emphasis in probabilistic category learning. Computational Brain \& Behavior, 3, 129-152.

Shepard, R. N. (1987). Toward a universal law of generalization for psychological science. Science, 237, 1317-1323.

Shondrick, S. J., Dinh, J. E., \& Lord, R. G. (2010). Developments in implicit leadership theory and cognitive science: Applications to improving measurement and understanding alternatives to hierarchical leadership. The Leadership Quarterly, 21, 959-978.

Simonton, D. K. (1994). Greatness: Who makes history and why. Guilford Press

Sluss, D. M., \& Ashforth, B. E. (2007). Relational Identity and Identification: Defining Ourselves Through Work Relationships. The Academy of Management Review, 32, 932.

Steffens, N. K., Haslam, S. A., Reicher, S. D., Platow, M. J., Fransen, K., Yang, J., Ryan, M. K., Jetten, J., Peters, K., \& Boen, F. (2014). Leadership as social identity management: Introducing the Identity Leadership Inventory (ILI) to assess and validate a four-dimensional model. The Leadership Quarterly, 25, 1001-1024.

Steffens, N. K., Haslam, S. A., Ryan, M. K., \& Kessler, T. (2013). Leader performance and prototypicality: Their inter-relationship and impact on leaders' identity entrepreneurship. European Journal of Social Psychology, 43, 606-613.

Steffens, N. K., Munt, K. A., van Knippenberg, D., Platow, M. J., \& Haslam, S. A. (2020). Advancing the social identity theory of leadership: A meta-analytic review of leader group prototypicality. Organizational Psychology Review.

Tajfel, H., \& Turner, J. C. (1979). An integrative theory of intergroup conflict. In W. G. Austin, \& S. Worchel (Eds.), The social psychology of intergroup relations (pp. 3347). Brooks/Cole.

Tenenbaum, J. B. \& Griffiths, T. L. (2001). Generalization, similarity, and Bayesian inference. Behavioral \& Brain Sciences, 24, 629-641. 
Todorov, A., Mandisodza, A. N., Goren, A., \& Hall, C. C. (2005). Inferences of competence from faces predict election outcomes. Science, 308, 1623-1626.

Turner, J. C. (1982). Towards a redefinition of the social group. In H. Tajfel (Ed.), Social identity and intergroup relations (pp. 15-40). Cambridge University Press.

Turner, J. C. (1991). Social influence. Open University Press.

Turner, J. C. \& Haslam, S. A. (2001). Social identity, organizations, and leadership. In M. E. Turner (Ed.), Applied social research. Groups at work: Theory and research, (pp. 2565). Erlbaum.

Turner, J. C., Hogg, M. A., Oakes, P. J., Reicher, S. D., \& Wetherell, M. S. (1987). Rediscovering the social group: A self-categorization theory. Basil Blackwell.

Tversky, A. (1977). Features of similarity. Psychological Review, 84, 327-352.

van Dick, R. (2001). Identification in organizational contexts: Linking theory and research from social and organizational psychology. International Journal of Management Reviews, 3, 265-283.

van Dick, R., Grojean, M. W., Christ, O., \& Wieseke, J. (2006). Identity and the extra mile: Relationships between organizational identification and organizational citizenship behaviour. British Journal of Management, 17, 283-301.

van Dick, R. \& Kerschreiter, R. (2015).The social identity approach to effective leadership: An overview and some ideas on cross-cultural generalizability. Frontiers of Business Research in China, 10, 363-384.

van Dick, R., Kerschreiter, R., Steffens, N. K., Akfirat, S.A., Dumont, K., et al. (2018). Identity leadership going global: Validation of the identity leadership inventory across 20 countries. Journal of Occupational and Organizational Psychology, 91, 697-728.

van Knippenberg, D. (2000). Work motivation and performance: A social identity perspective. Applied Psychology, 49, 357-371.

van Knippenberg, D. (2011). Embodying who we are: Leader group prototypicality and leadership effectiveness. The Leadership Quarterly, 22, 1078-1091.

van Knippenberg, D., \& Hogg, M. A. (2003). A social identity model of leadership effectiveness in organizations. Research in Organizational Behavior, 25, 243-295.

van Knippenberg, B., \& van Knippenberg, D. (2005). Leader self-sacrifice and leadership effectiveness: The moderating role of leader prototypicality. Journal of Applied Psychology, 90, 25-37.

Van Quaquebeke, N. V., Graf, M. M., \& Eckloff, T. (2014). What do leaders have to live up to? Contrasting the effects of central tendency versus ideal-based leader prototypes in leader categorization processes. Leadership, 10, 191-217.

Vanpaemel, W. \& Storms, G. (2008). In search of abstraction: the varying abstraction model of categorization. Psychonomic Bulletin \& Review, 15, 732-749. 
Voorspoels, W., Vanpaemel, W., \& Storms, G. (2011). A formal ideal-based account of typicality. Psychonomic Bulletin \& Review, 18, 1006-1014.

Walumbwa, F. O., Avolio, B. J., Gardner, W. L., Wernsing, T. S., \& Peterson, S. J. (2008). Authentic leadership: Development and validation of a theory-based measure. Journal of Management, 34, 89-126.

Zaki, S. R., Nosofsky, R. M., Stanton, R. D., \& Cohen, A. L. (2003). Prototype and Exemplar Accounts of Category Learning and Attentional Allocation: A Reassessment. Journal of Experimental Psychology: Learning, Memory, \& Cognition, 29, 1160-1173. 


\section{Appendix}

\section{Preliminary Screening of Rating Responses}

To confirm that participants were sufficiently engaged with the rating task and considering their responses, we examined the distribution of survey completion times. The median finishing time was 10 minutes and 29 seconds, which was inconsistent with the idea that participants were failing to consider their responses to the questions. We also examined the number of fast finishing times, as this would provide a clearer indicator of inattentive responding. Only 33 participants completed the survey in under 5 minutes, which is $3.7 \%$ of the final analyzed sample, leading us to believe that the vast majority of participants were sufficiently engaged with the task.

We also checked the proportion of stimuli for which the same rating was provided across all stimulus attributes — but allowed for different ratings across different stimuli (e.g., responding "1” across all dimensions for Barack Obama, and responding "7” across all dimensions for Abraham Lincoln; note we did not just consider the extreme ends of the rating scale, and checked for this flat pattern of responding for any rating value). We found that $7.78 \%$ of all responses conformed to this flat response pattern, with only 3 participants out of 902 responding in this way to each stimulus they were presented with. Given the fairly low rate of "flat" responding and the fact that many of those ratings could reflect genuine responses, we surmise that low-effort responding was fairly uncommon and participants were, on the whole, quite engaged with the task.

\section{Distributions of Leader Competence and Presidential Effectiveness Rating Data}

For each stimulus, we summarized rating data for our two main dependent variables of interest, leader competence and US presidential effectiveness in the main text (Figures 4 
and 5). To give a sense of the variability in ratings for each stimulus, we report histograms summarizing these data in Figures A1 and A2.

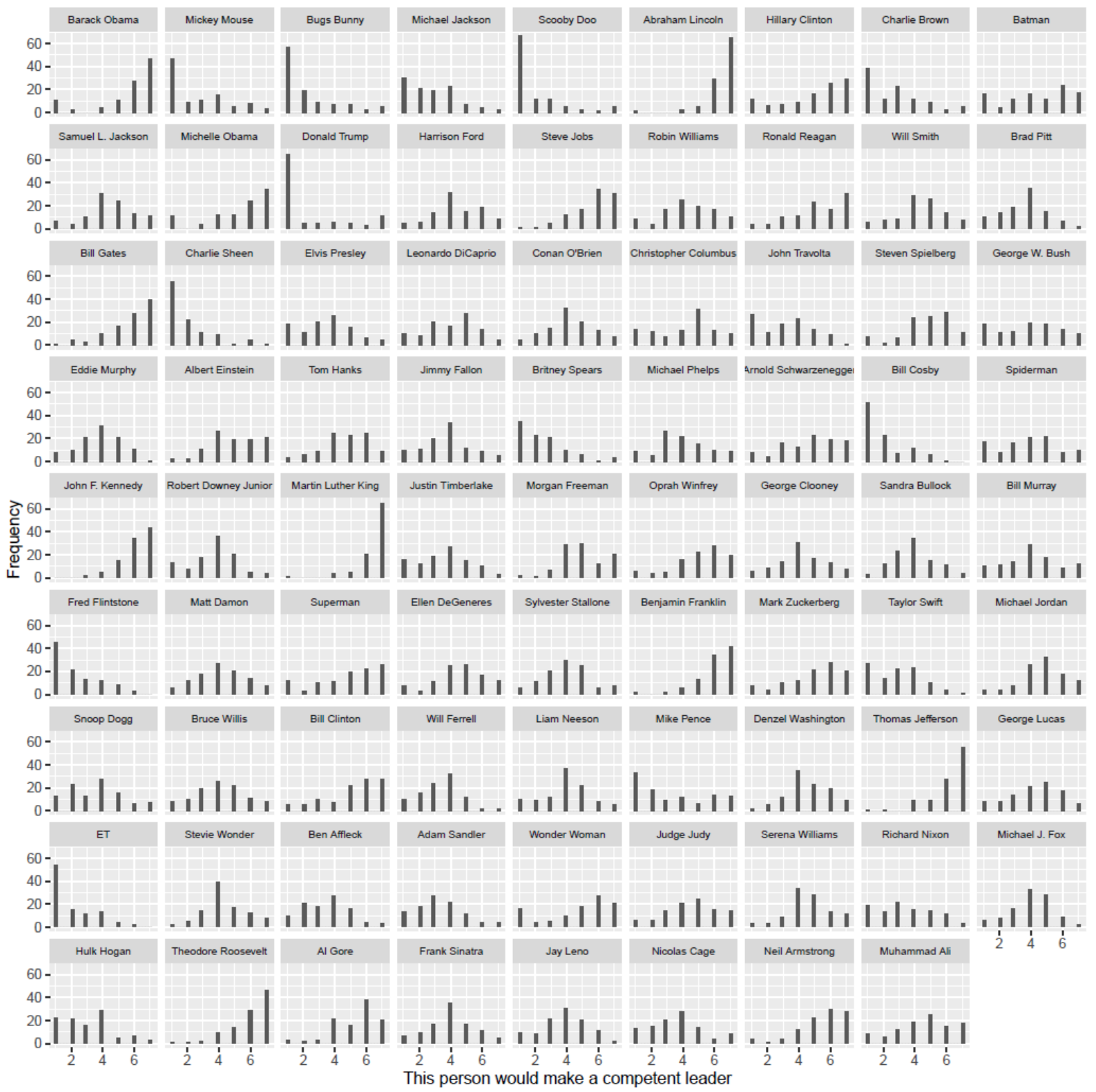

Figure 10. Histograms showing distributions of ratings for each stimulus in terms of overall leader competence. 


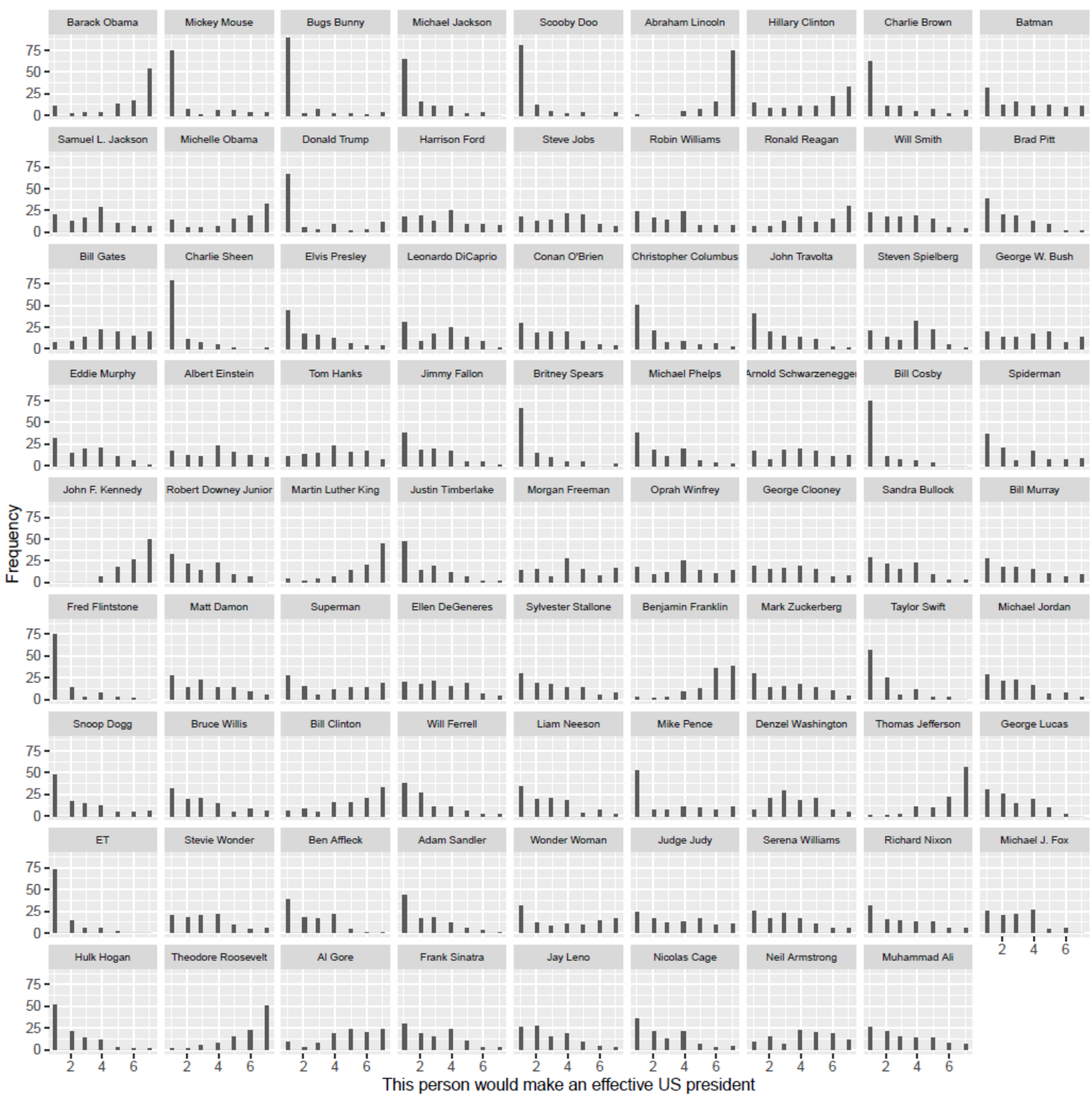

Figure A2. Histograms showing distributions of ratings for each stimulus identity in terms of perceived presidential effectiveness.

\section{Alternative Modeling Results Using a Euclidean Distance Metric}

The modeling analyses reported in the main text were based on a so-called city-block metric for computing psychological distance (Equation 2 in the main text). The city-block metric is most appropriate when the stimulus dimensions are psychologically separable (Garner, 1974). With psychologically separable dimensions, information about one 
dimension need not influence the perception of information along another dimension. Given that our dimensional structure was theoretically motivated by the Steffens et al. (2014) attribute structure, we believe the assumption of psychological separability is appropriate. In the interest of caution, however, we also modeled the data using a Euclidean distance metric, which is defined as,

$$
d_{i j}=\sqrt{\sum_{m=1}^{M} w_{m}\left|x_{i m}-x_{j m}\right|^{2}}
$$

where $m$ indexes different dimensions, and $i$ and $j$ describe the stimulus and a psychological representation (e.g., an exemplar), respectively. Fitting the models using the Euclidean distance metric resulted in near-identical parameter estimates for the GCM, and similar parameter estimates for the prototype model. In the latter case, there are some differences in the attention weights across dimensions, but the overall quality of fit was very similar to applications with a city-block metric. For both models, the patterns of predictions were very similarly, and led to the same inferences and conclusions presented in the main text. Fit indices and best-fitting parameter estimates for the models are reported in Table A1. 
Table A1. Model fit statistics and best-fitting parameter estimates for fits to the Leader Competence and Presidential Effectiveness data sets using a Euclidean distance metric. Parameter estimates for attention weights and response bias are normalized.

\begin{tabular}{|c|c|c|c|c|c|c|c|c|c|c|c|}
\hline Data Set & $\ln L$ & $R^{2}$ & Embodying & Advancing & Creating & Embedding & Authenticity & Identification & $c$ & $\gamma$ & $b_{+}$ \\
\hline $\begin{array}{l}\text { Prototype: } \\
\text { Leader } \\
\text { Competence }\end{array}$ & 190.99 & 0.74 & 0.00 & 0.26 & 0.00 & 0.31 & 0.17 & 0.26 & 18.50 & - & 0.52 \\
\hline $\begin{array}{l}\text { Prototype: } \\
\text { US } \\
\text { Presidential } \\
\text { Effectiveness }\end{array}$ & 172.11 & 0.66 & 0.00 & 0.36 & 0.00 & 0.42 & 0.00 & 0.22 & 17.47 & - & 0.36 \\
\hline $\begin{array}{l}\text { GCM: } \\
\text { Leader } \\
\text { Competence }\end{array}$ & 222.51 & 0.88 & 0.00 & 0.33 & 0.00 & 0.47 & 0.11 & 0.09 & 8.28 & 2.54 & 0.47 \\
\hline $\begin{array}{l}\text { GCM: US } \\
\text { Presidential } \\
\text { Effectiveness }\end{array}$ & 223.59 & 0.91 & 0.00 & 0.50 & 0.00 & 0.45 & 0.00 & 0.05 & 31.86 & 1.39 & 0.33 \\
\hline
\end{tabular}




\section{Modeling Results Including Data From Participants Who Failed the Attention Check}

The modeling results reported in the main text excluded participants who failed the attention check question, but otherwise provided complete survey data. Table A2 summarizes the results of the main modeling analyses, showing that the results are nearly identical to those discussed in the main text. None of the theoretical conclusions are altered if our inferences were based on data including participants who failed the attention check. 
Table A2. Model fit statistics and best-fitting parameter estimates for fits to the Leader Competence and Presidential Effectiveness data sets including data from the $N=21$ participants who failed the attention check, but otherwise provided complete survey data. Parameter estimates for attention weights and response bias are normalized.

\begin{tabular}{|c|c|c|c|c|c|c|c|c|c|c|c|}
\hline Data Set & $\ln L$ & $R^{2}$ & Embodying & Advancing & Creating & Embedding & Authenticity & Identification & $c$ & $\gamma$ & $b_{+}$ \\
\hline $\begin{array}{l}\text { Prototype: } \\
\text { Leader } \\
\text { Competence }\end{array}$ & 188.55 & 0.72 & 0.19 & 0.23 & 0.00 & 0.37 & 0.07 & 0.15 & 9.01 & - & 0.52 \\
\hline $\begin{array}{l}\text { Prototype: } \\
\text { US } \\
\text { Presidential } \\
\text { Effectiveness }\end{array}$ & 172.02 & 0.66 & 0.00 & 0.38 & 0.00 & 0.42 & 0.00 & 0.20 & 9.88 & - & 0.36 \\
\hline $\begin{array}{l}\text { GCM: } \\
\text { Leader } \\
\text { Competence }\end{array}$ & 220.16 & 0.87 & 0.00 & 0.34 & 0.00 & 0.46 & 0.11 & 0.09 & 7.93 & 2.62 & 0.47 \\
\hline $\begin{array}{l}\text { GCM: US } \\
\text { Presidential } \\
\text { Effectiveness }\end{array}$ & 223.60 & 0.91 & 0.00 & 0.51 & 0.00 & 0.43 & 0.00 & 0.06 & 45.20 & 1.32 & 0.33 \\
\hline
\end{tabular}

Note. $\operatorname{lnL}=$ Log Likelihood; Identification = Identification with this Individual. 


\section{Modeling Results Excluding Fictional and Historical Figures from the Stimulus Set}

We also considered a reduced version of the data set, which excluded fictional and historical figures from the set of exemplars used for modeling purposes. We defined historical figures as any individual who had died over 100 years ago, which resulted in removal of Abraham Lincoln, Christopher Columbus, Benjamin Franklin, Thomas Jefferson, and Theodore Roosevelt from the stimulus set. We also removed the fictional characters, Batman, Bugs Bunny, Charlie Brown, ET, Fred Flintstone, Mickey Mouse, Scooby Doo, Spiderman, Superman, and Wonder Woman from the stimulus set. Overall, this resulted in the exclusion of 15 stimuli, leaving $N=65$ stimuli which served as exemplars (for the GCM) and the basis for forming prototypes (for the prototype model). Fit statistics and parameter estimates for the models, as applied to the Leader Competence and Presidential Effectiveness data sets are shown in Table A3. 
Table A3. Model fit statistics and best-fitting parameter estimates for fits to the Leader Competence and Presidential Effectiveness data sets excluding fictional and historical figures from the stimulus set. Parameter estimates for attention weights and response bias are normalized.

\begin{tabular}{|c|c|c|c|c|c|c|c|c|c|c|c|}
\hline Data Set & $\ln L$ & $R^{2}$ & Embodying & Advancing & Creating & Embedding & Authenticity & Identification & $c$ & $\gamma$ & $b_{+}$ \\
\hline $\begin{array}{l}\text { Prototype: } \\
\text { Leader } \\
\text { Competence }\end{array}$ & 205.63 & 0.70 & 0.26 & 0.00 & 0.00 & 0.54 & 0.00 & 0.20 & 9.94 & - & 0.53 \\
\hline $\begin{array}{l}\text { Prototype: } \\
\text { US } \\
\text { Presidential } \\
\text { Effectiveness }\end{array}$ & 188.74 & 0.60 & 0.00 & 0.32 & 0.00 & 0.42 & 0.00 & 0.26 & 10.61 & - & 0.36 \\
\hline $\begin{array}{l}\text { GCM: } \\
\text { Leader } \\
\text { Competence }\end{array}$ & 251.05 & 0.90 & 0.00 & 0.17 & 0.00 & 0.50 & 0.00 & 0.33 & 50.00 & 1.20 & 0.50 \\
\hline $\begin{array}{l}\text { GCM: US } \\
\text { Presidential } \\
\text { Effectiveness }\end{array}$ & 229.67 & 0.86 & 0.00 & 0.51 & 0.00 & 0.43 & 0.00 & 0.06 & 43.74 & 1.38 & 0.32 \\
\hline
\end{tabular}

Note. $\ln \mathrm{L}=$ Log Likelihood; Identification = Identification with this Individual. 
The theoretical conclusions based on the modeling of this reduced stimulus set are the same as our main analysis. That is, the GCM provides a superior fit to both data sets in terms of AIC (i.e., -486.10 vs. -397.26 for the Leader Competence data; -443.34 vs. -363.48 for the Presidential Effectiveness data). The prototype model remains unable to capture the highest and lowest ratings, failing for precisely the same reasons as in our main analysis. We do note that some of the parameter estimates for the GCM are slightly different with the reduced stimulus set — the specificity parameter, $c$, takes on a higher value for the Leader Competence data. We conjecture that removal of the fictional and historical figures trimmed the data set of especially good/bad leader exemplars, depriving the model of potentially useful reference points for judging the leadership capacity of certain individuals (e.g., an individual who is highly similar to Abraham Lincoln would likely be judged to be a very competent leader, whereas one who is highly similar to Bugs Bunny would likely be judged to be a very poor leader). The five lowest-rated leaders for both Leader Competence and Presidential Effectiveness included Bugs Bunny, ET, and Scooby Doo. By contrast, the five highest-rated leaders for Leader Competence included Abraham Lincoln, Benjamin Franklin, and Thomas Jefferson. These same three individuals were among the five highest-rated leaders on Presidential Effectivness, with Theodore Roosevelt also included in the top five. Overall, we conclude that our main analysis, supporting the GCM over the prototype model, is higly robust to changes in the underlying stimulus set, and our conclusions were not dependent on including fictional and historical figures in the stimulus set. 